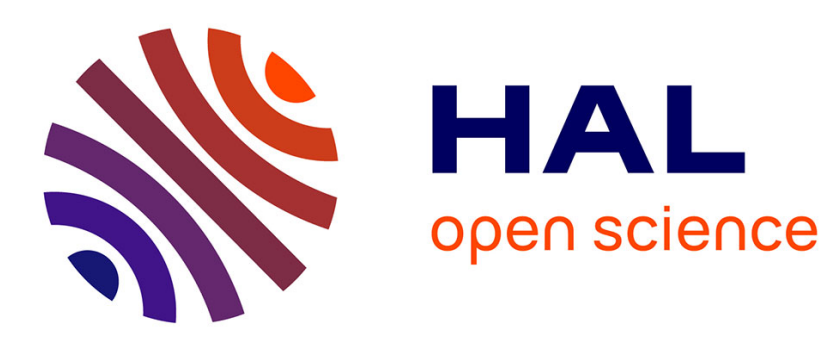

\title{
Elastocapillarity: When Surface Tension Deforms Elastic Solids
}

José Bico, Etienne Reyssat, Benoit Roman

\section{To cite this version:}

José Bico, Etienne Reyssat, Benoit Roman. Elastocapillarity: When Surface Tension Deforms Elastic Solids. Annual Review of Fluid Mechanics, 2018, 50 (1), pp.629-659. 10.1146/annurev-fluid-122316050130 . hal-02359002

\section{HAL Id: hal-02359002 https://hal.science/hal-02359002}

Submitted on 12 Nov 2019

HAL is a multi-disciplinary open access archive for the deposit and dissemination of scientific research documents, whether they are published or not. The documents may come from teaching and research institutions in France or abroad, or from public or private research centers.
L'archive ouverte pluridisciplinaire $\mathbf{H A L}$, est destinée au dépôt et à la diffusion de documents scientifiques de niveau recherche, publiés ou non, émanant des établissements d'enseignement et de recherche français ou étrangers, des laboratoires publics ou privés. 


\section{Elastocapillarity: When surface tension deforms elastic solids}

\section{José Bico, Étienne Reyssat and Benoît Roman}

Physique et Mécanique des Milieux Hétérogènes (PMMH),

ESPC-Paris, PSL Research University, Sorbonne Universit, Univ. Paris Diderot, 10 rue Vauquelin, 75005 Paris France

email: jose.bico@espci.fr, etienne.reyssat@espci.fr, benoit.roman@espci.fr

Xxxx. Xxx. Xxx. Xxx. YYYY. AA:1-33

This article's doi:

$10.1146 /(($ please add article doi $))$

Copyright (C) YYYY by Annual Reviews.

All rights reserved

\section{Keywords}

capillarity, elasticity, interfacial flows, fluid-structure interaction

Abstract

Although negligible at large scales, capillary forces may become dominant for submillimetric objects. Surface tension is usually associated with the spherical shape of small droplets and bubbles, wetting phenomena, imbibition or the motion of insects at the surface of water. However, beyond liquid interfaces, capillary forces can also deform solid bodies in their bulk as observed in recent experiments with very soft gels. Capillary interactions, which are responsible for the cohesion of sand castles, can also bend slender structures and induce the bundling of arrays of fibres. Thin sheets can finally spontaneously wrap liquid droplets within the limit of the constraints dictated by differential geometry.

The aim of this review is to describe the different scaling parameters and characteristic lengths involved in "elastocapillarity". We focus successively on three main configurations:

- 3D, deformations induced in bulk solids

- 1D, bending and bundling of rod-like structures

- $2 \mathrm{D}$, bending and stretching of thin sheets

Although each configuration would deserve a detailed review, we hope our broad description will provide a general view on elastocapillarity. 
Capillary length:

$\ell_{c}=\sqrt{\frac{\gamma}{\rho g}}$

\section{INTRODUCTION}

Fluid structure interactions are traditionally considered at large scales: wind dynamic pressure blows up the sails of a ship, whereas hydrostatic pressure tends to deform the hull. Rain may complicate manoeuvring on the deck, but the capillary forces responsible for the spherical shape of the droplets have a negligible impact at this scale. At small scales (more exactly, at small Reynolds numbers), interactions between viscous forces and soft structures are important in the locomotion of micro-organisms and can induce deformations of cells or blood vessels, as recently reviewed (Duprat and Stone, 2015). It is well known that surface tension forces are associated with the motion of aquatic insects at the surface of water, capillary rise and wetting (de Gennes et al., 2004). In terms of length scale, capillarity becomes dominant over gravity below the capillary length $\ell_{c}=\sqrt{\gamma / \rho g}$. Beyond liquid interfaces, can surface tension (or surface stress) also deform solids?

Recent technological advances in microfabrication and the development of very soft materials have motivated numerous studies of the coupling between surface tension and elasticity. In this review, we propose to explore the currently blooming field of elastocapillarity. We propose to develop three main configurations characterised by a specific dimension:

- 3D: at which scale may surface tension deform bulk solids?

- 1D: how do bundles spontaneously appear in a brush removed from a liquid bath?

- 2D: can a thin sheet spontaneously wrap a droplet?

This review does not pretend to be exhaustive and interested readers are invited to read other more specific reviews as a complementary approach. A recent review from Style el al. present the most recent advances in the deformation of a bulk solid by surface tension (Style et al., 2017). Snoeijer also discusses the coupling of lubrication flow, surface tension and elasticity with the illustration of the familiar scraper coating process (Snoeijer, 2016). Many stimulating reviews have been dedicated to capillary self-assembly of small objects by capillary forces (Boncheva and Whitesides, 2005, Mastrangeli et al., 2009) and to the collapse (Maboudian, 1997) or folding (Syms et al., 2003, Leong et al., 2010, Crane et al., 2013) of engineered microstructures. In the more specific context of surface engineering, impressive achievements have been realised with arrays of nanorods or carbon nanotubes assembled through capillary interactions (De Volder and Hart, 2013, Tawfick et al., 2016). From an academic prospective, the bundling of arrays fibres has finally been described in different reviews (Roman and Bico, 2010, Duprat and Stone, 2015). The aim of the present review is to provide a broad view of elastocapillarity. The description of each configuration is limited to main scaling laws, with the hope they would bring useful physical insights. More specific details will be found in the cited literature.

\section{CAPILLARITY AND BULK ELASTICITY}

\subsection{The elasto-capillary length}

Surface tension is mostly thought to be of interest when dealing with liquids. However, solids are also subject to interfacial stresses which may induce deformations. Nevertheless, a key difference between simple liquids and solids is the ability of solid interfaces to sustain finite strains. As a consequence, surface stresses in solids are strain dependant. The surface stress $\Upsilon$ and surface energy $\gamma$ of a solid are related through the Shuttleworth equation,

2 Bico, Reyssat, \&3 Roman 
which in its simplest form reads:

$$
\Upsilon=\gamma+\frac{\mathrm{d} \gamma}{\mathrm{d} \varepsilon}
$$

where $\varepsilon$ is the interfacial strain (Andreotti and Snoeijer, 2016).

On which scale may surface stresses induce deformations in elastic solids? While trying to measure the surface tension of solids (metals or ionic crystals), Nicolson has shown that the characteristic length scale at which surface stresses become important is defined by the competition of surface forces with bulk elastic stresses (M. M. Nicolson, 1955). The characteristic stress resulting from surface tension, given by the classical Laplace law, is of order $\Upsilon / L$, where $L$ is the size of the solid sample. Such stresses may induce elastic deformations in the bulk of the solid. The induced strain becomes of order one when stresses reach the shear modulus $G$ of the solid. The effect of surface stresses is thus important on structures whose characteristic size is smaller than the "shear elastocapillary length":

$$
\ell_{S}=\Upsilon / G
$$

For usual materials such as glass, surface tension is of order $250 \mathrm{mN} / \mathrm{m}$, for an elastic modulus $G \simeq 20 \mathrm{GPa}$, so that $\ell_{s} \sim 10 \mathrm{pm}$ is below atomic scales. Similarly, in the case of copper, $\Upsilon \sim 1.8 \mathrm{~N} / \mathrm{m}$ and $G \sim 50 \mathrm{GPa}$ and we obtain $\ell_{S} \sim 40 \mathrm{pm}$. For such hard solids, precise X-ray measurements may detect small variations in the lattice parameter as a function of the size of the sample, but for most purposes, surface effects remain undetected. In the past decades, there has been a lot of interest for much softer materials such as elastomers, gels or biological tissues. These materials are highly compliant, with typical values of $G \sim 0.1-1000 \mathrm{kPa}$ and $\Upsilon \sim 0.01-0.1 \mathrm{~N} / \mathrm{m}$. For this class of materials, $\ell_{S}$ is at least $10 \mathrm{~nm}$ and may be as large as $1 \mathrm{~mm}$. Such deformations may provide ways to measure the surface tension of a solid-fluid interface, which remains poorly documented. Capillaryinduced deformations also constitute a useful tool to probe the mechanics of biological cells as recently reported (Campàs et al., 2013).

2.1.1. Softening sharp solids. As it is the case for liquid-gas interfaces, the surface tension of solid tends to smooth interfaces with high curvatures. For instance, a number of experimental and theoretical studies motivated by contact printing discuss the rounding off of the sharp edges of printing stamps made of soft elastomeric materials (Hui et al., 2002). These stamps are initially formed into a stiff mold. When peeled off the master, the topography of the replica tends to be smoothed out by surface stresses. A corner initially at right angle is for instance rounded off with a radius $R \sim \Upsilon / G$. Similarly, the edges of a square based prism made of a very soft gel $(G=35 \mathrm{kPa})$ are softened by surface tension (Mora et al., 2013). Mora and Pomeau also derive analytically the shape of angular solids in the asymptotic regimes of very shallow or extremely sharp wedges (Mora and Pomeau, 2015). The softening of the edges of a solid also induce more global deformations at the large scale of a slender object, which may be used to shape materials or monitor surface stresses. For instance, ribbons with asymmetric triangular cross-section take the shape of helices under the action of surface tension (Pham et al., 2013).

Periodic grooves or ripples patterns on the surface of very soft gels are also altered significantly (Paretkar et al., 2014). The sharpest features are erased, or equivalently, high spatial Fourier frequencies are attenuated under the action of surface tension. The relative change $\Delta A$ in the amplitude $A$ of the grooves of wavelength $\lambda$ is of order $\Delta A / A \sim \Upsilon / G \lambda \sim$ $\ell_{S} / \lambda$. Moreover, the surface tension of these gels of polydimethylsiloxane (PDMS), may

shear elasto-capillary length: $\ell_{S}=\Upsilon / G$ 


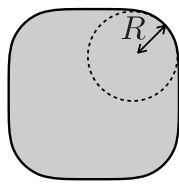

(a)
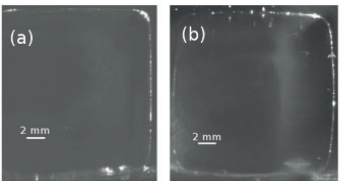

(b)

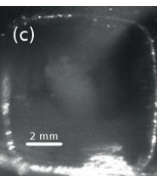

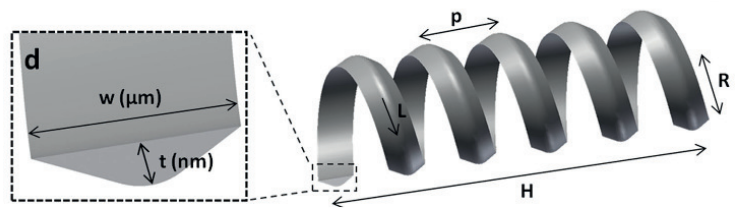

(c)

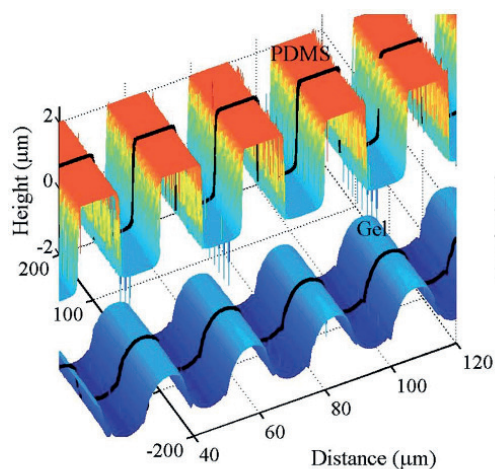

(d)

Figure 1

(a) Surface tension rounds off the corner of soft solids with a radius $R$ on the order of $\ell_{s}$ (adapted from (Hui et al., 2002)). (b) The initially square cross-section of soft PDMS gels is rounded off when dipped in toluene (illustration from (Mora and Pomeau, 2015)). The shear modulus of the gel is $125 \mathrm{~Pa}$ (left), $60 \mathrm{~Pa}$ (center), $60 \mathrm{~Pa}$ (right). The effect is more pronounced for smaller and softer objects. (c) Ribbons with asymetric cross-section deform under the action of solid surface tension. The edges with different sharpness are deformed unequally, leading to the curling of the ribbon into a helical shape (Pham et al., 2013). (d) The sharp texture of the interface of a soft solid smoothens when removed from its mold (Paretkar et al., 2014).

be modified by exposure to UV light, leading to some degree of control of the surface tension (Jagota et al., 2012).

Interfacial textures may finally emerge from the Biot instability, where creasing patterns result from compression along the surface of a soft solid. The threshold for creasing has recently be found to depend on the surface tension of the solid (Mora et al., 2011, Chen et al., 2012).

2.1.2. Rayleigh-Plateau instability. A paradigm of capillary induced instability is the classical Rayleigh-Plateau instability: the liquid stream falling from a faucet breaks up into a collection of droplets with overall lower surface to volume ratio (de Gennes et al., 2004). An analogous phenomenon occurs in the case of very soft solid cylinders.

Upon drying, a filament of acrylamid gel develops a tensed skin, which results in the growth of a peristaltic instability illustred in Figure 2a. (Matsuo and Tanaka, 1992, Barrière et al., 1996). Even in the absence of a macroscopic skin, a strand of very soft agar gel released in toluene develops undulations along its axis (Mora et al., 2010). Axial modulations of the radius are found to develop if the gel/toluene surface tension exceeds $\Upsilon_{c} \sim G \rho_{0}$, where $\rho_{0}$ is the radius of the undeformed cylinder and $G$ is the shear modulus of the gel (figure 2). The instability develops preferentially large wavelengths. Related instabilities occur for fluid or solid cylinders embedded in another compliant matrix with various geometries, as has been studied theoretically and numerically (Henann and Bertoldi, 2014, Taffetani and Ciarletta, 2015, Xuan and Biggins, 2016).

2.1.3. Modification of the apparent elastic properties of multiphase solids. Since interfaces couple to bulk mechanical properties of soft materials, we expect soft multiphase

4 Bico, Reyssat, ES Roman 


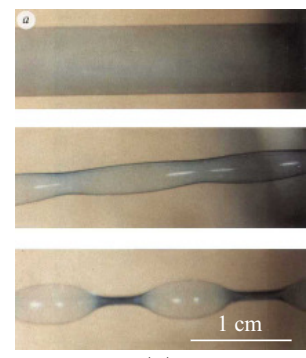

(a)

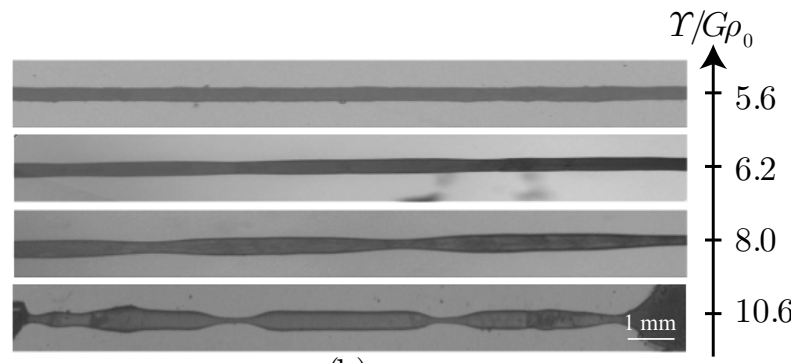

(b)

Figure 2

(a) Upon drying, cylinders of acrylamide gels undergo a pearling instability. Adapted from (Matsuo and Tanaka, 1992). (b) When dipped in toluene, cylinders of soft agar gels develop a peristaltic instability driven by surface stresses. Adapted from (Mora et al., 2010)

solids to display anomalous properties. According to the classical results from Eshelby, an elastic matrix containing gas or liquid inclusions should appear softer than a plain material (Eshelby, 1957). Conversely, inclusions with large shear modulus result in an overall stiffer material. Extending these results to include the effects of surface tension yields counter-intuitive results. Indeed, deforming small inclusions tends to increase their area. Surface stresses thus oppose these deformations. As a consequence, a solid with soft inclusions may appear stiffer than a plain specimen. Ducloué et al. show that elastic porous materials do not follow Eshelby's prediction (Ducloué et al., 2014): while air bubbles tend to weaken oil-in-water emulsions, the softening is lower than expected. Style et al. even show experimentally that liquid droplets smaller than $\ell_{S}$ stiffen the material in which they are embedded (Style et al., 2015). This effect has also been recently explored through molecular dynamics simulations (Liang et al., 2016).

As surface effects dominate at small scales, elastocapillary phenomena are expected to be relevant for the field of nanotechnologies, which has been expanding in the past decade. Understanding the mechanical properties of micro- and nanoscale devices is crucial for applications. Different studies conducted with metallic wires of sub-micrometer diameter reveal that their apparent stiffness deviates from predictions based on bulk values of the Young modulus. For instance, apparent Young's moduli of silver or lead deduced from bending tests on nanowires depends on the geometry of the wires (Cuenot et al., 2004). For a wire of radius $r$ and length $L$, surface effects indeed dominate bulk contributions in the bending stiffness when $r^{3} / L^{2}<\ell_{S}$.

\subsection{Wetting soft solids}

In the previous configurations surface stresses result from a single solid fluid interface. However, a large number of situations also involve contact lines where three different phases meet (eg a sessile droplet). In this section, we focus on the deformations at the vicinity of this singular contact line.

2.2.1. Neumann and Young's laws. Before describing the possible deformations induced by a liquid drop on a soft solid susbrate, we recall the two classical laws of static wetting in the respective cases of liquid or rigid solid substrates as sketched in Figure 3 
(de Gennes et al., 2004). Consider first a drop of liquid (phase 1) floating at the surface of a bath of immiscible liquid (phase 2) in another fluid (phase 3). At equilibrium, the vector balance of the tensions of the three interfaces applies at the contact line:

$$
\Upsilon_{12} \mathbf{t}_{12}+\Upsilon_{13} \mathbf{t}_{13}+\Upsilon_{23} \mathbf{t}_{23}=\mathbf{0}
$$

where $\Upsilon_{i j}$ is the interfacial tension between phases $i$ and $j$ and $\mathbf{t}_{i j}$ is the vector pointing along the $i / j$ interface (Figure 3(a)). Equation 3, known as Neumann equation, sets the angles between the interfaces at the contact line.

The second classical result of wetting, the Young and Dupré equation, corresponds to the case of a liquid droplet deposited on a flat rigid substrate (Figure 3(b)). Balancing horizontal forces prescribes the value of the Young contact angle $\theta$ :

$$
\Upsilon_{13} \cos \theta=\Upsilon_{23}-\Upsilon_{12}
$$

Although both equations 3 and 4 have been successfully used to describe the wetting properties of liquids, these laws also constitute a subject of debate in the framework of wetting or adhesion on deformable solids. Early works from Lester (Lester, 1961) and Rusanov (Rusanov, 1978) question the validity of Young's law in the case of wetting of very soft materials. This question has recently been blooming again as macroscopic values of $\ell_{S}$ can be obtained with very soft gels (Marchand et al., 2012, Weijs et al., 2013, Weijs et al., 2014, Style et al., 2017).

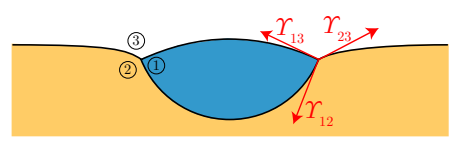

(a)

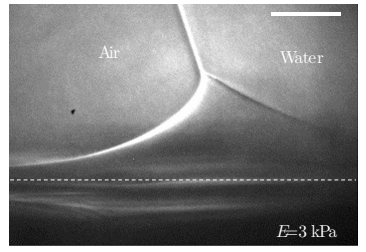

(d)

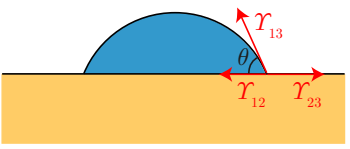

(b)

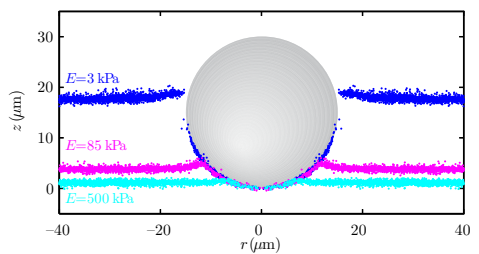

(e)

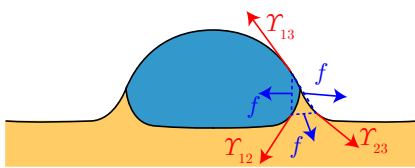

(c)

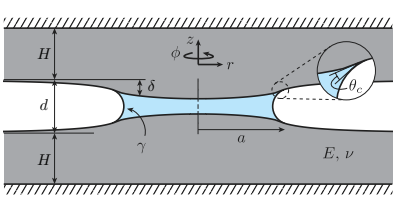

(f)

Figure 3

(a) Neumann balance of interfacial tensions at a contact line: the vector sum of surface tensions at the contact line vanishes. (b) Drop on a stiff solid: the contact angle is given by Young's law, which expresses the balance of horizontal projections of interfacial stresses. (c) On a soft solid, the upward component of the liquid surface tension is balanced by elastic stresses in the substrate, leading to the formation of a wetting ridge at the contact line. (d) Asymmetric wetting ridge induced by a water drop at the surface of a very soft silicone gel. The scale bar represents $5 \mu \mathrm{m}$. Adapted from (Park et al., 2014). (e) Profile of silicone gel layers deformed upon adhesion of silica particles of radius $15 \mu \mathrm{m}$ (adapted from (Style et al., 2013c)). (f) The shallow gap of thickness $d$ separating two layers of soft material may be squeezed by the negative Laplace pressure in a liquid bridge connecting both walls. Adapted from (Wexler et al., 2014). 
2.2.2. Static wetting and adhesion. Consider a liquid drop deposited on the initially horizontal surface of a soft material. If we neglect gravity, the drop takes the shape of a spherical cap, and is characterised by its Young contact angle $\theta$. However, Young's law seems to only provide an incomplete description of the situation, as it overlooks the vertical components of surface tensions. The liquid-gas surface tension indeed has a component normal to the solid surface which must be balanced. The wetting of a soft solid thus results in the formation of a ridge along the contact line of the drop (Figure 3(c), (d)). Shanahan was a pioneer in describing theoretically the profile of the wetting ridge (Shanahan, 1987) and more recent studies are inspired by his early work (Jerison et al., 2011, Limat, 2012). The shape and size of the ridge is set by a balance of surface stresses and elastic forces in the bulk of the soft solid, so that the characteristic size of the deformed region is of the order of the elasto-capillary length $\ell_{S}$. While the expected size of the ridge is very small for usual elastomers (sub-micrometric for $G \sim 1 \mathrm{MPa}$ ), the shape of wetting ridges has recently been measured experimentally in the context of wetting of soft gels (Pericet-Camara et al., 2008). Jerison et al. report accurate measurements of the displacement field of wetting ridges in the vicinity of the contact line formed by a water drop deposited on an elastic substrate (Jerison et al., 2011). Their theoretical model shows excellent agreement with experimentally measured displacement fields, for the particular case of a drop contact angle $\theta=\pi / 2$. One key ingredient is the introduction of the finite thickness of the layer of gel, which regularises the divergence of the height of the ridge emerging in early models. In the general configuration, $\theta \neq \pi / 2$ and displacements may also occur along the solid surface on which the drop rests. Tangential forces are applied on the solid and observed through the formation of asymetric wetting ridges (Fig. 3d) (Das et al., 2011, Park et al., 2014).

The validity of classical laws of wetting by Neumann and Young (equations 3 and 4) in the wetting of soft solids is discussed by several authors (Das et al., 2011, Style and Dufresne, 2012, Style et al., 2013a). Measuring the contact angles of drops of various sizes is one way to shed light on this problem. In particular, the contact angle of small drops on soft solids satisfies Neumann's criterion, while larger drops obey Young's law (Style et al., 2013a, Style et al., 2017). The crossover between both regimes occurs for drops of radius $R \sim \ell_{S}$ : at small scales, surface tension effects dominate over bulk forces. More generally, Neumann's balance of surface tensions applies in the close vicinity of a contact line, while Young's law is recovered at scales larger than $\ell_{S}$.

The balance of surface tensions and bulk forces can be generalised to any combination of three phases, one of whom at least is a soft solid. This is for instance the case (see Figure 3(e)) of the adhesion of stiff (soft) particles on soft (stiff) substrates (Salez et al., 2013, Style et al., 2013c, Andreotti et al., 2016) or the shape of soft particles floating at the interface between two liquids (Mehrabian et al., 2016). In any configuration, deformations occur in the compliant phase on a scale $\ell_{S}$ around the triple line.

In a different geometry, a bridge of wetting liquid connecting two opposing soft walls also provides capillary adhesion due to its negative internal Laplace pressure. Consider two facing layers of soft material of thickness $H$ deposited on stiff grounds and separated by a gap $d$ (Figure 3(f)). If $\delta$ is the displacement of the surface of the soft layer, the induced strain scales as $\delta / H$ (we assume that the width of the liquid bridge is large in comparison with $H$ ). The corresponding stress $E \delta / H$ balances Laplace pressure $\gamma / d$, leading to $\delta \sim(\gamma / E)(H / d)$. As a consequence, the gap of initial thickness $d$ between these compliant walls may be squeezed if $d^{2} / H<\ell_{S}$ (Wexler et al., 2014). 
2.2.3. Dynamical effects. The motion of a contact line at velocity $V$ over a stiff substrate is usually described by the balance of viscous and interfacial stresses through the capillary number $C a=\eta V / \gamma$ where $\eta$ and $\gamma$ are the viscosity and surface tension of the liquid (de Gennes et al., 2004). The deformation of elastic substrates by droplets strongly impacts the phenomena of dynamic wetting. Indeed, moving contact lines induce dissipation in the wetting ridges which adds up to the usual viscous forces in the liquid. In early works, Shanahan describes experimentally and theoretically how viscous dissipation in the induced ridge may drastically slow down the spreading mechanism of a liquid drop (Shanahan, 1988). One signature of this effect is that the velocity of a drop sliding under gravity on a soft substrate is governed by the viscoelastic properties of the solid and may become independent of the liquid viscosity (Carré et al., 1996, Long et al., 1996). On soft solids, analogs of classical dynamic wetting laws involve a "solid capillary number" that compares surface tension to viscoelastic dissipation in the bulk of the substrate (Karpitschka et al., 2015).

A wetting ridge at a contact line constitutes a local and reversible roughness which enhances contact angle hysteresis on soft surfaces (Extrand and Kumagai, 1996). As a result, rich contact line dynamics emerge on viscoelastic substrates: lines may for instance move following a stick-slip motion in connection with the dynamics of the elastic ridge itself (Kajiya et al., 2013, Karpitschka et al., 2015). Impact dynamics are also significantly affected by viscoelastic dissipation in the substrate. The retraction of a spread droplet after impact is for instance significantly hindered (Alizadeh et al., 2013) and "it is harder to splash on soft solids" (Howland et al., 2016).

The coupling of capillary forces to the deformations of elastic substrates may lead to interesting new applications. Gradients in the elastic properties of the substrate may for instance become the driving force for the motion of droplets along a soft substrate (Style et al., 2013b), a process coined as durotaxis by analogy with cell motility. One may also hope for more efficient ways to harness transfer or dew collection, as the condensation processes on soft substrates have been shown to be more efficient than on stiff surfaces (Sokuler et al., 2010).

\section{CAPILLARY-INDUCED BENDING OF RODS}

In the previous section, we described how a drop of water may deform a bulk material. Nevertheless, the elastocapillary length scale involved remains relatively small for usual situations. However, we know from our everyday experience that a macroscopic brush with unaligned bristles tend to form an elegant bundle when it is removed from a bath of water. In this section, we propose to explore the bending deformation induced to slender structures by capillary forces as encountered in a wide variety of natural or engineered systems.

We first focus on the buckling of a single fibre by a liquid interface and derive a characteristic length scale based on the coupling between bending and capillary forces. The single fibre configuration is extended to the case of a brush or, more generally, to arrays of fibres. We then describe how van der Waals interactions replace capillary forces when the liquid evaporates and lead to "stiction". We finally present other elastic modes of deformation, involving stretching, twisting that are also relevant to micro-engineering applications.

8 Bico, Reyssat, \& Roman 


\subsection{A rod in a drop: capillary buckling}

As a simple academic configuration, consider a thin rod trapped inside a droplet of wetting liquid in the absence of gravity or any other external field (Figure 4a). If the volume of liquid is decreased (e.g. upon evaporation), should we expect the confined rod to pierce the interface or, conversely, to match the spherical boundaries of its liquid container?

Piercing the interface would result into a compressive force $F=p \gamma \cos \theta$, where $p$ is the perimeter of a cross section of the rod, $\gamma$ the liquid surface tension and $\theta$ the Young contact angle of the liquid on the rod (de Gennes et al., 2004). For the sake of simplicity we assume that the liquid perfectly wets the $\operatorname{rod}(\theta=0)$. The general case with finite contact angle has also been addressed (Andreotti et al., 2011). As a consequence, we expect the simply supported rod to buckle if $F$ reaches the critical force derived by Euler, $F_{c}=\pi^{2} E I / L^{2}$, where $L$ is the length of the rod, $I$ the second moment of inertia and $E$ the Young modulus of the material (Audoly and Pomeau, 2010). In other words, the rod should buckle if its length exceeds the critical length $L_{c}=\pi \ell_{B}$, where the bending length $\ell_{B}$ is given by (Cohen and Mahadevan, 2003):

$$
\ell_{B}=\sqrt{\frac{E I}{p \gamma}}
$$

In the case of a cylindrical rod of radius $r$, we obtain $p=2 \pi r, I=(\pi / 4) E r^{4}$ and $\ell_{B}=$ $\sqrt{E r^{3} / 8 \gamma}$. Thin lamellae of width $w$ and thickness $t$ with $t \ll w$ are also common in academic studies as well as in practical situations (Neukirch et al., 2007). In this geometry, we obtain similarly $p=2 w$ and $I=E t^{3} w / 12\left(1-\nu^{2}\right)$, where $\nu$ is the Poisson coefficient of the material, which leads to $\ell_{B}=\sqrt{E t^{3} / 24\left(1-\nu^{2}\right) \gamma}$.

(a)

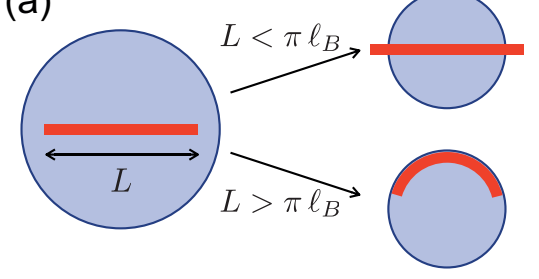

(b)

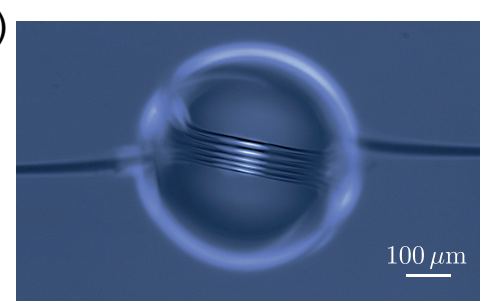

\section{Figure 4}

a) Rod trapped in a droplet of wetting liquid: decreasing the volume of liquid eventually results in a compressive load of the rod by the surface tension of the deformed interface. If the length of the rod exceeds a critical capillary-bending length scale $\ell_{B}$, the rod buckles. b) Coiling of a rod inside a droplet (Schulman et al., 2017).

This capillary-induced buckling mechanism has for instance been proposed to explain the formation of rings of carbon nanotubes inside cavitation bubbles (Martel et al., 1999). If the length of the flexible rod is large in comparison with the perimeter of the droplet, the rod may eventually form a coil into the droplet as illustrated in Figure $4 \mathrm{~b}$ (Roman and Bico, 2010, Schulman et al., 2017). In a different field, capillary-induced coiling is also crucial for the capture of preys by some orb-weaver spiders. Although the radial threads of spider nets are renowned for their amazing mechanical properties, radial threads also display unique damping properties that prevent impacting preys from bouncing off the web (Vollrath and Edmonds, 1989). The damping mechanism was only recently evidenced (Elettro et al., 2016). The spiralling thread spun by the spider is

Capillary-bending length:

$\ell_{B}=\sqrt{\frac{E r^{3}}{8 \gamma}}$

for a cylinder,

$\ell_{B}=\sqrt{\frac{E t^{3}}{24\left(1-\nu^{2}\right) \gamma}}$

for a lamella. 
actually significantly longer than its apparent length. The excess of length is stored in coils formed into minute droplets deposited along the thread by the spider. In contrast with an elastic string were the pulling force increases linearly with strain, the force required to extend the thread is almost constant (equal to $F_{c}$ if viscous forces are neglected). After being stretched, the coil reforms slowly due to the high viscosity of the droplets, which ensures the efficient damping of an impacting prey.

\subsection{Elastocapillary rackets: measuring the capillary-bending length scale}

How can $\ell_{B}$ be measured? Determining accurately the critical buckling length would not be convenient. However, measuring the deflection induced by a lateral load could be more efficient.

3.2.1. Scaling law. A simple technique consists in bending a ribbon into the shape of a racket and assembling both ends with some wetting liquid (Figure 5a). At equilibrium, both extremities meet parallely. The shape of the lamella follows Euler elastica mechanics (Audoly and Pomeau, 2010) and is universal for such boundary conditions. However, the bending elastic energy stored in the lamella depends on its size:

$$
\mathcal{U}_{b} \sim \frac{E I}{L^{2}} L
$$

where the length $L$ of the bent part of the ribbon also gives the scale for its curvature. Increasing the size of the racket reduces the elastic energy but also results in a cost of surface energy. In the case of flexible lamellae of width $w$, the surface energy of the system is proportional to:

$$
\mathcal{U}_{s} \sim \gamma w\left(L-L_{t}\right)
$$

where $L_{t}$ is the total length of the lamella. Minimising the global energy of the system thus sets the size of the racket (Cohen and Mahadevan, 2003, Py et al., 2007a):

$$
L \sim \ell_{B}
$$

A simple way of assessing $\ell_{B}$ thus consists in measuring the width $d$ of the racket. The numerical prefactor has to be determined numerically: $d=1.26 \ell_{B}$. The same scaling can be generalised to rods although the derivation of the capillary energy slightly depends on the volume of liquid in the meniscus connecting the facing extremities (Py et al., 2007a).

3.2.2. Boundary conditions. The Euler elastica equation has to be solved in order to predict the exact shape of the racket (Audoly and Pomeau, 2010). For a beam of bending stiffness $E I$, the evolution of the angle $\alpha$ made by the tangent $\mathbf{t}$ to the lamella with the horizontal is set by a moment balance along a portion $\mathrm{d} s$ of the beam:

$$
E I \frac{\mathrm{d}^{2} \alpha}{\mathrm{d} s^{2}} \mathbf{e}_{\mathbf{z}}+\mathbf{t} \times \mathbf{R}=\mathbf{0}
$$

where $\mathbf{R}$ is the constant vectorial tension acting on the beam, $\mathbf{e}_{\mathbf{z}}$ is the direction perpendicular to the plane and $s$ the curvilinear coordinate (Figure $5 \mathrm{~b}$ ). This equation can be solved through standard shooting methods for given boundary conditions at both extremities. Although the position and the slope of the tips are trivial, the balance between capillary forces

Bico, Reyssat, 83 Roman 

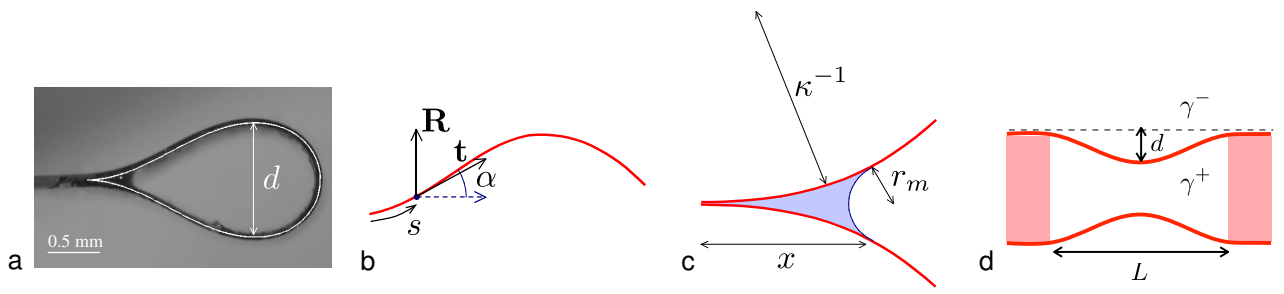

Figure 5

(a) Elastocapillary racket: the opposite strands of a stip looped on itself are maintained by a liquid meniscus. The shape of the loop is compared with a numerical solution of Euler elastica equation (Py et al., 2007b). The width $d$ of the racket provides a measure of $\ell_{B}$. (b) Coordinates used in the elastica equation. (c) Close-up of the capillary bridge in the vicinity of the contact point of the strands. (d) Floating flexible frame deflected by a contrast in surface tension. The surface pressure $\Pi=\gamma^{+}-\gamma^{-}$can be inferred from monitoring the amplitide of deflection $d$.

and bending stiffness results into a specific boundary condition a the contact point of the merged extremities: the initial curvature $\kappa=\mathrm{d} \alpha / \mathrm{d} s$ is proportional to $1 / \ell_{B}$.

The precise derivation of the curvature jump at the contact point requires variational methods (Majidi and Adams, 2009). However, this initial curvature can be viewed as a simple moment balance (Figure 5c). If we note $x$ the lateral extension of the liquid meniscus at the junction between both strands of the racket, its radius of curvature is proportional to $r_{m} \sim \kappa x^{2}$ (we assume $x \ll \kappa^{-1}$ ). The resulting Laplace pressure is thus on the order of $P \sim \gamma / r_{m}$, which leads to a force $F \sim P x w$ where $w$ is the width of the beam. The corresponding moment $F x \sim \gamma w / \kappa$ balances the bending moment $E I \kappa$, which finally leads to:

$$
\kappa^{-1} \sim \ell_{B}
$$

The numerical prefactor depends on the exact configuration. In the case of a pair of merging lamellea, we find $\kappa^{-1}=\ell_{B} / \sqrt{2}$ (Roman and Bico, 2010). In the case of rods, the induced curvature is independent of the quantity of liquid and can even be applied to dry adhesion (Majidi and Adams, 2009).

3.2.3. Capillary induced deflection of flexible frames. A contrast in surface tension between each side of a flexible frame floating at the surface of water can also induce a deflection of the frame. Measuring the amplitude of this deflection constitutes an elegant way to assess surface pressures on a Langmuir trough (Hu et al., 2003, Zell et al., 2010).

Consider a rectangular frame of length $L$ with two rigid sides floating at the surface of water. Adding surfactant molecules outside the frame results in a contrast in surface tension $\Delta \gamma$, which induces a deflection of the edges of the frame of amplitude $d$ (Figure $5 \mathrm{~d}$ ). The corresponding elastic energy scales as $E I L(d / L)^{2}$ while the gain in surface energy is proportional to $\Delta \gamma d L$. As a result, the amplitude varies linearly with the surface pressure:

$$
d \sim \frac{L^{2}}{E I} \Delta \gamma
$$

The same concept has recently been used to estimate the local surface tension of a soap film (Adami and Caps, 2015). 
As a conclusion of this section, the capillary-bending length prescribes the characteristic curvature surface tension may induce to a slender structure. An alternative way to define this length scale would be to deposit the slender structure on a cylinder of radius $R$ coated with a wetting liquid. The structure spontaneously wraps the cylinder for $R>\ell_{B}$. In the opposite situation, the structure remains undeformed.

So far, we focused on a single rod. In the next section we propose to describe the bundling of arrays of filaments induced by capillary forces.

\subsection{Elastocapillary self-assembly}

3.3.1. Bundles: from engineered systems to model experiments. In the previous section we described how an isolated slender structure is prone to buckle under the action of surface tension if its length exceeds the capillary bending length $\ell_{B}$. However, a large number of practical situations involve arrays of structures (Figure 6). For instance, the possible collapse of micro-structures is an important constraint in the design of Micro-Electro-Mechanical Systems (MEMS). Indeed, standard lithography processes involve the evaporation of a solvent, which generates liquid bridges that can be fatal for minute microstructures (Tas et al., 1996, Abe and Reed, 1996, Maboudian, 1997). In material science, many works on carbon nanotube "forests" have reported the formation of pyramidal bundles (Lau et al., 2003) or cellular patterns (Chakrapani et al., 2004, Correa-Duarte et al., 2004) after the evaporation of a solvent. Similar structures are also commonly observed with arrays of micro-pillars obtained with soft polymers (Duan and Berggren, 2010, Hu et al., 2015, Kim et al., 2016). Intriguing twisted bundles may even be observed (Pokroy et al., 2009, Kang et al., 2010). In material science, beautiful works have recently been devoted to "elasto-capillary engineering" of filamentary materials (Tawfick et al., 2016). Elegant nanotube "gardens" are for instance produced by combining the controlled growth of carbon nanotubes on silicon substrates and capillary bundling (García et al., 2007, De Volder et al., 2010, De Volder et al., 2011). Harnessing capillary forces to build complex structures paves the way for interesting applications such as field emission (Wang et al., 2016), enhanced molecule detection (Hu et al., 2010) or surfaces with tunable colour (Chandra and Yang, 2010).

Such capillary-induced bundling is finally not limited to engineered systems but is also frequent in Nature. The barbules of bird feathers may for instance form fatal clumps when contaminated by oil (Duprat et al., 2012). At a smaller scale, the adhesive setae from some insect species may also form bundles (Gernay et al., 2016).

What are the physical ingredients driving elastocapillary bundling? Model experiments conducted with macroscopic brushes show that bundling involves a cascade of pairing reminiscent of coalescence mechanisms (Bico et al., 2004, Kim and Mahadevan, 2006, Py et al., 2007a): as the brush is withdrawn from a bath of wetting liquid, pairs of smaller bundles merge into large bundles that later merge larger bundles (Figure 7a). However, an alternative mechanism involving simultaneous bundling is also relevant during the drying of arrays of dense pillars. In the following sections, we propose to present both mechanisms.

3.3.2. Merging a pair of beams. As an elementary model configuration, consider two parallel plates separated by a distance $d$, small in comparison with the capillary length $\ell_{c}$. 


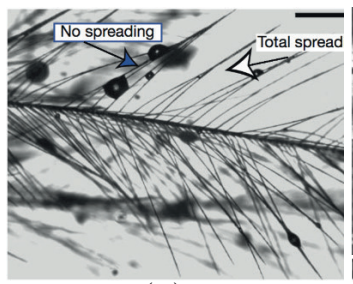

(a)

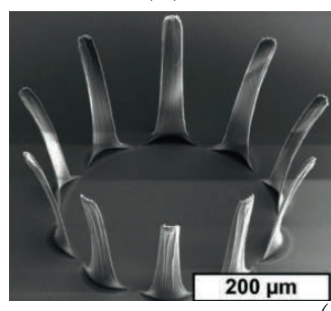

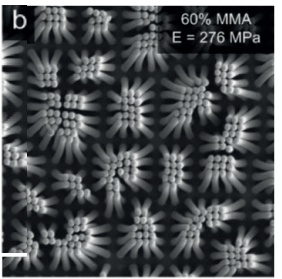

(b)
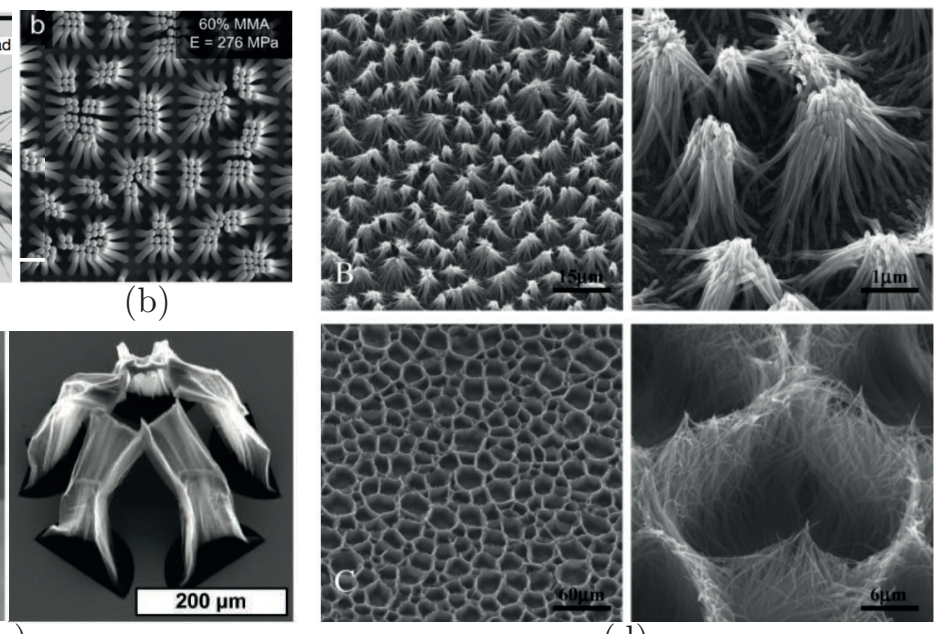

(d)

\section{Figure 6}

Examples of capillary induced bending and eventually bundling of array of slender structures: (a) oil-contaminated barbules of a feather (Duprat et al., 2012), (b) array of bundled soft polymeric pillars (Chandra and Yang, 2009), (c) and (d) self-assembled arrays of carbon nanotubes into "nanogardens" (De Volder et al., 2010) or into pyramidal bundles or cellular patterns (Correa-Duarte et al., 2004).

If the lamellae were rigid, Jurin's law would dictate the equilibrium height of rise as $h_{J}=2 \gamma / \rho g d$ (de Gennes et al., 2004). However, the low pressure in the rising liquid tends to bring both lamellae together, which results in a thinner gap and to a higher equilibrium height. The rising process eventually stops when the cost in elastic energy corresponding to the bending of the lamellea compensates the gain in surface energy. If the lamellae are long enough, the liquid reaches an equilibrium height at a fixed distance $L_{\text {stick }}$ from the clamping base of the lamellae (Figure $7 \mathrm{~b}$ ). This distance is readily derived with the same energy argument used in the previous section to describe the size of a racket.

In the limit $L_{\text {stick }} \gg d$, the typical curvature of the bent lamella $d / L_{\text {stick }}^{2}$ results into the elastic energy:

$$
\mathcal{U}_{b} \sim E I\left(\frac{d}{L_{\text {stick }}^{2}}\right)^{2} L_{\text {stick }}
$$

As in the case of the racket, the surface energy reads:

$$
\mathcal{U}_{s} \sim \gamma w\left(L_{s t i c k}-L_{t}\right)
$$

for lamellae of total length $L_{t}$ and width $w$. Minimising the global energy $\mathcal{U}_{b}+\mathcal{U}_{s}$ with respect to $L_{\text {stick }}$ provides the sticking length:

$$
L_{\text {stick }} \sim \ell_{B}^{1 / 2} d^{1 / 2}
$$

as validated experimentally for both array of lamellae (Bico et al., 2004, Kim and Mahadevan, 2006) or of cylindrical rods (Py et al., 2007a). 

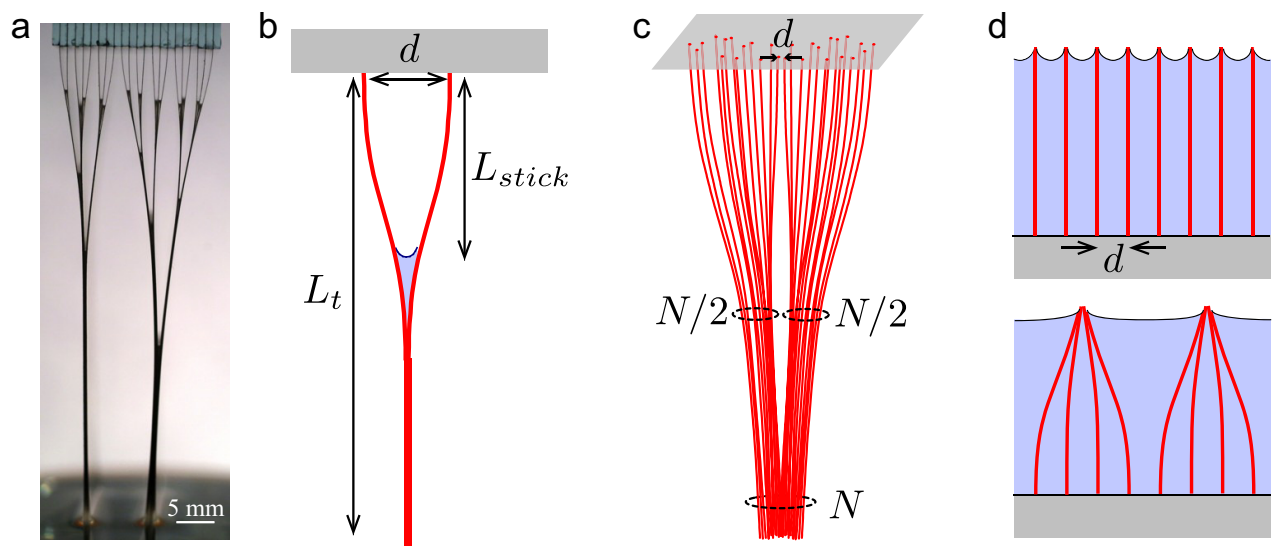

Figure 7

(a) Bundling mechanism involving a cascade of pairing as a brush of long fibres is withdrawn from a bath of wetting liquid (Bico et al., 2004). (b) A pair of parallel beams of total lenght $L_{t}$ and separated by a spacing $d$ adhere up to a distance $L_{\text {stick }}$ from their clamping base when put in contact with a wetting liquid. (c) Bundling sequence of a 2D array of fibres: two bundles of average size $N / 2$ merge into a large bundle of size $N$. (d) Alternative mechanism involving a single step as an initially immersed array of fibres is progressively dried. Sketch adapted from (Tawfick et al., 2016).

3.3.3. Dynamics. In terms of dynamics, different time scales can be defined as a function of the stiffness of the lamellea. In the case of rigid lamellae, the interplay between the viscous flow driven by surface tension and the limiting hydrostatic pressure leads to a typical rising time (de Gennes et al., 2004):

$$
\tau_{r} \sim \frac{\eta \gamma}{(\rho g)^{2} d^{3}} \sim \frac{\eta}{\gamma} \frac{\ell_{c}^{2}}{d^{3}}
$$

In the opposite case of long flexible lamellae, the dynamics is dominated by the closure of the gap induced by the low pressure in the rising liquid. In this situation, the resulting visco-elastic time scale is given by (Duprat et al., 2011):

$$
\tau_{v e} \sim \frac{\eta}{\gamma} \frac{L_{t}^{6}}{d^{3} \ell_{B}^{2}}
$$

Nevertheless this relation is not universal and we expect elasto-capillary rise between circular rods to follow different time scales. The rising liquid can indeed flow along the curved corners formed along contacting rods.

Another time scale has also been proposed to describe a configuration where the liquid flows from the clamp to the tip (Aristoff et al., 2011, Taroni and Vella, 2012): $\tau_{b} \sim \eta \ell_{B} / \gamma$. A future comprehensive study of elastocapillary dynamics involved in the different practical situations would be highly valuable.

3.3.4. Elastocapillary coalescence. The pairing mechanism presented in the previous section can be adapted to larger scales. A bundle composed of $N$ beams indeed results from the grouping of two smaller bundles of average size $N / 2$. Both smaller bundles may be considered as effective beams with a stiffness multiplied by $N / 2$ (assuming that the presence 
of a thin layer of fluid lubricates the relative sliding of the beams) and a separating distance that is also amplified (Figure 7 ). The effective distance is simply $N d / 2$ for a simple linear array and proportional to $N^{1 / 2} d$ in the case of a 2-dimensional lattice. Inputting this effective stiffness and distance in Equation 14, we obtain the merging length for a bundle of size $N L_{\text {stick }}(N) \sim N^{3 / 4} \ell_{B}^{1 / 2} d^{1 / 2}$ for a linear array. In the case of $2 \mathrm{D}$ lattice, the scaling is slightly different, $L_{\text {stick }}(N) \sim N^{3 / 8} \ell_{B}^{1 / 2} d^{1 / 2}$. The inversion of this relation prescribes the maximum size $N_{\max }$ of a $2 \mathrm{D}$ bundle composed of flexible elements of length $L_{t}$ :

$$
N_{\text {max }} \sim \frac{L_{t}^{8 / 3}}{\ell_{B}^{4 / 3} d^{4 / 3}}
$$

in agreement with experimental data obtained with model brushes (Py et al., 2007a).

3.3.5. Simultaneous bundling. Although the previous model is relevant for long brushes withdrawn from a liquid bath, alternative scenarios have been developed to describe the drying of $2 \mathrm{D}$ arrays of dense pillars initially immersed in a volatile liquid. In this approach, bundles are formed in a single step when the interface of the liquid reaches the top of the pillars (Figure $7 \mathrm{~d}$ ). For pillars of radius $r$, deforming the interface corresponds to a cost in surface energy on the order $\gamma r^{2}$ per pillar. As a consequence, pillars mutually attract through lateral capillary forces (Kralchevsky and Nagayama, 2000, Chandra and Yang, 2009). However, forming a bundle also results into bending elastic energy. For a bundle containing $N$ pillars, the average deflection is proportional to $N^{1 / 2} d$. As a consequence, the elastic energy per bent pillar scales as $E r^{4} N d^{2} / L^{3}$. Comparing surface and bending energies, we obtain the following scaling for the maximum size of a bundle:

$$
N_{\max } \sim\left(L / L_{s}\right)^{3} \quad \text { with } L_{s}=\left(\frac{E r^{2} d^{2}}{\gamma}\right)^{1 / 3}
$$

This scenario is consistent with experimental data obtained with Si nanorods (Zhao and Fan, 2006) or epoxy micropillars (Chandra and Yang, 2010) corresponding to pillar densities $r / d \sim 0.2$.

3.3.6. Size distribution. Although both bundling mechanisms provide a maximum size $N_{\max }$ for the self-assembled clusters, wide size distributions are observed in experiments (Boudaoud et al., 2007). Nevertheless, distributions can be rescaled by $N_{\max }$, and their actual shape rely on dynamics. In the cascade mechanism for instance, the first step consists in forming pairs. However, about one third of the flexible structures remain statistically unpaired as their first neighbours are already paired. Successive associations can later on lead to bundles of size $3,4,5 \ldots$ and finally to any size below $N_{\max }$. Different approaches have been proposed to describe this distribution from crude mean field (Boudaoud et al., 2007) to more sophisticated dynamical models, such as arrays of rigid plates with elastic hinges (Bernardino and Dietrich, 2012, Wei and Mahadevan, 2014) or idealised brushes of parallel lamellae (Singh et al., 2014, Hadjittofis et al., 2016). The comparison of the two last references with experimental data is particularly convincing. Nevertheless, a comprehensive study of the size distribution in the case of 2D arrays of pillars still remains to be conducted both experimentally and theoretically. 
Combining buckling and bundling mechanisms may also explain qualitatively different morphologies observed experimentally such as pyramidal bundles or cellular patterns. Indeed, isolated pillars or small bundles may buckle and collapse on the substrate, while wider bundles may resist and form the walls of the observed cells (Chiodi et al., 2010, Tawfick et al., 2016). However, further work would be necessary to confirm quantitatively such scenario.

3.3.7. Stiction. Although capillary forces can induce the formation of bundles, we might expect elastic microstructures to recover their initial shape once the liquid has totally evaporated. However, collapsed structures generally remain permanently deformed due to attractive molecular forces, which is a major issue for Micro-Electro-Mechanical Systems (MEMS) (Tas et al., 1996, Abe and Reed, 1996, Maboudian, 1997) or microcontact printing (Hui et al., 2002, Sharp et al., 2004). Van der Waals surface energy $W_{a}$ involved in contact adhesion is indeed of the same order of magnitude as the surface tension. In the case of contact between flat surfaces, the relations between elasticity and surface tension can be adapted by simply replacing $\gamma$ by $W_{a}$ (DelRio et al., 2005). When cylindrical rods are involved, the actual contact width $a$ depends on a balance between adhesion energy and material stiffness, $a \sim\left(\frac{W_{a}}{E} r^{2}\right)^{1 / 3}$ (Chaudhury et al., 1996). As a consequence $\gamma$ should be replaced by $W_{a}^{4 / 3} /(E r)^{1 / 3}$ (Roca-Cusachs et al., 2005, Kim et al., 2016).

\subsection{Collapsing tubes and channels}

In the previous sections, we showed how capillary forces may induce the collapse of simple slender structures such as rods or lamellae. However, similar effects are also observed in tubular geometries. For instance, tongues of hummingbirds have a peculiar gutter shape which spontaneously closes by capillarity when in contact with a liquid. Nectar is then safely transported through the self-assembled straw (Rico-Guevara and Rubega, 2011).

The deformation of compliant channels induced by capillary forces may however be tragic in other situations. The collapse of lung airways of premature babies is a major issue which has motivated numerous academic studies (Grotberg and Jensen, 2004). If we consider a flexible tube internally liquid-lined, capillary energy is lowered if the tube collapses, as the liquid-vapour surface is reduced (Figure 8a). The mechanism involves a snap-through instablility and occurs when surface tension forces overcome the rigidity of the cross-section of the tube (Hazel and Heil, 2003). As a crude approximation, the problem is similar to the deformation of flexible frames presented in Section 3.2.3. A tube of radius $R$ and wall thichness $t$ is expected to buckle and eventually collapse for $R \gg \ell_{B} \sim \sqrt{E t^{3} / \gamma}$ (note that the relevant rigidity is based on the deformation of the cross-section and not in the bending of the tube along its length as in bundling).

From an engineering prospective, soft microfluidic channels may also deform under Laplace pressure drop, which may enhance the flow velocity (Anoop and Sen, 2015) or capillary rise (Cambau et al., 2011). In an ideal situation, a microfluidic channel may be viewed as a rigid groove of width $w$ and depth $d$ covered with a plate of thickness $t$ (Figure 8b). The deflection $\delta$ induced by a pressure drop of order $\gamma / d$ is readily estimated from a torque balance. The capillary moment per unit length scales as $\gamma w^{2} / d$, while the restoring moment from the bent plate corresponding to a typical curvature $\delta / w^{2}$ is proportional to $E t^{3} \delta / w^{2}$. 
At equilibrium, the induced deflection should thus follow:

$$
\delta \sim \frac{w^{4}}{d \ell_{B}^{2}}
$$

The closing condition is thus similar to the adhesion of wet lamellae: $w \sim \ell_{b}^{1 / 2} d^{1 / 2}$, in agreement with experiments conducted with a model setup (Cambau et al., 2011). The actual problem is however more complex as stretching of the wall of the channel is usually also involved.
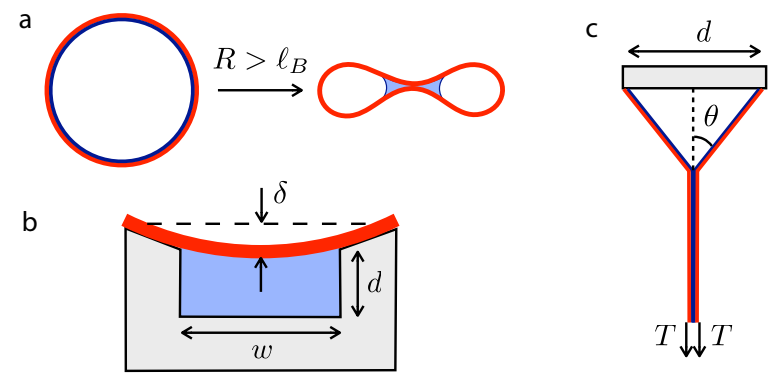

Figure 8

(a) Possible collapse of a flexible tube of radius $R>\ell_{B}$ lined with a layer of liquid. (b) Deflection of a channel covered with a flexible lid. (c) Adhesion of a pair of lamellae submitted to a tensile load $T$ in the ideal case where the lamellae do not have any bending rigidity but are unstretchable.

\subsection{Capillarity against tension}

Deformations induced by capillary forces are not limited to bending modes. The capillary adhesion of a pair of parallel rods or ribbons submitted to a tensile force has for instance recently been investigated (Duprat and Protiere, 2015, Legrain et al., 2016).

As a model experiment, consider a pair of vertical lamellea of length $L$ clamped at their upper extremities with a separating distance $d$ and each submitted to a constant tension $T$ per unit width, e.g. by hanging weights. We assume that the lamellae do not have any bending rigidity but are unstretchable. Upon introduction of some wetting liquid inside the spacing, the lower parts of the lamellae join at a distance $L_{\text {stick }}$ from the clamp and form an angle $\theta$ with the vertical as sketched in Figure $8 \mathrm{c}\left(\tan \theta=d / 2 L_{\text {stick }}\right)$. The interfacial energy (per unit width) gained by a lamella by adhering to its neighbour is $\mathcal{U}_{\gamma}=\gamma\left(L-L_{\text {stick }} / \cos \theta\right)$. As a result of sticking, the bottom point of the lamella lifts by an amount $\delta=d(1-\cos \theta) / 2 \sin \theta$, inducing a work $W_{T}=-T \delta$ of the tension force. The equilibrium $\mathrm{Y}$ shape formed by the adhered lamellae is found by maximizing $W_{T}+\mathcal{U}_{\gamma}$ with respect to $\theta$, yielding:

$$
\cos \theta=\frac{T}{T+\gamma}
$$

which is reminiscent of the equation defining Young contact angle. Note that the tension is not necessarily constant and may depend on $\theta$. For instance, merging lamellae of length $L$ and thickness $t$ clamped at both ends without any prestrain induces a progressively increasing tension. Indeed, the corresponding strain can be estimated from elementary 
geometry as $\varepsilon=\frac{1-\cos \theta}{\sin \theta} \frac{d}{L}$, which leads to the tension $T=E t \frac{1-\cos \theta}{\sin \theta} \frac{d}{L}$. In such a case, the adhesion angle $\theta$ is determined by solving the non-linear equation obtained by imputing the value of $T(\theta)$ in the expression for $\cos \theta$.

\section{DROPS AND ELASTIC SHEETS}

In the previous section, we have described how capillary forces may buckle or, more generally deflect a rod. Can these results be extended to plates? Bending a plate of thickness $h$ along a single direction involves the same bending elastic energy proportional to $h^{3}$ as in the case of a rod. However, we know that wrapping an orange with a sheet usually leads to complex folds and wrinkles. Indeed, bending simultaneously a sheet in two different directions also involves stretching (Audoly and Pomeau, 2010). Since stretching energy scales linearly with $h$, the cost of stretching is relatively very high in comparison with bending in the limit of small thicknesses. As a consequence, whenever possible, a thin sheet complies with geometrical constraints or mechanical load by pure bending, preserving the distances along its surface, with important consequences on elasto-capillary phenomena.

In this section we propose to first present the conditions for the spontaneous wrapping of a liquid droplet with a sheet. We then focus on the capillary adhesion of a thin sheet on a rigid substrate, as an illustration of how geometrical constraints may impose a finite stretching of the sheet. We finally describe the case of a small droplet deposited on a floating sheet and show how the stretching modulus of the plate becomes a relevant parameter, due to the tension induced by capillary forces.

\subsection{Capillary origami}

4.1.1. Folding hinges. Although complex 2D structures are commonly obtained through conventional lithography, building 3D micro-objects remains challenging. Capillary forces constitute a promising tool to self-assemble micro-objects in $2 \mathrm{D}$ and even in $3 \mathrm{D}$ (Boncheva and Whitesides, 2005, Mastrangeli et al., 2009). Impressive 3D structures have for instance been obtained by depositing a tiny droplet of water on flat templates where hinges have been previously designed (Legrain et al., 2014). When the droplet is in contact with the template, capillary forces acting at the contact line tend to pull the flaps, while the positive Laplace pressure tends to push the hinge away (Figure 9a). The global effect is a moment of the order of $\gamma L$ per unit width, where $L$ is the length of the flaps (we here assume that the radius of the droplet is comparable to the size of the structure). As a consequence, the hinge closes with angle $\alpha \sim \gamma L / C$ where $C$ is the rotational stiffness of the hinge per unit width. Surface tension forces may even be activated on demand using a small quantity of tin deposited on the hinges (Syms et al., 2003, Filipiak et al., 2009). As temperature is increased, the tin melts into a liquid droplet whose surface tension may fold the hinge (Figure 9a). Elegant sub-micrometre self-folding cases have been produced with such techniques (Cho et al., 2010).

4.1.2. Folding elastic plates. In a technologically simpler situation, a featureless plate (without hinges) may also fold with a very good reproducibility, but now involves distributed elastic bending of the plate. When a droplet of liquid is deposited on a flexible template (Py et al., 2007b), the torque induced by capillary forces tends to bend the plate into a shape that depends on the volume of liquid. The radius of the droplet is here compa-

Bico, Reyssat, 83 Roman 
a)

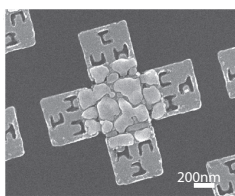

b)

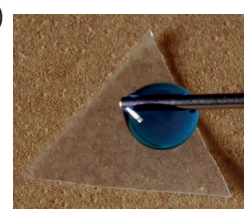

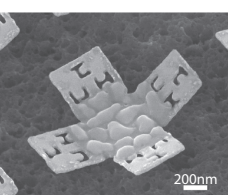

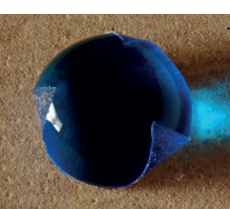

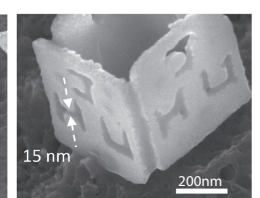

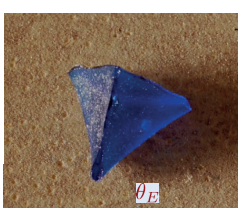

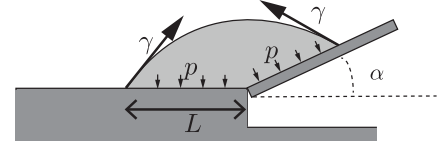

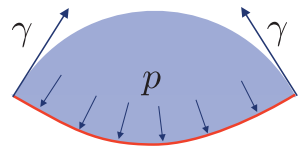

Figure 9

Folding elastic plates with surface tension : a) Capillary forces resulting from melting a thin layer of tin induce a moment and consequently, a rotation along the hinges at a submicrometre scale (pictures from (Cho et al., 2010)). b) A droplet deposited on a thin elastic sheet may bent the sheet to minimize the liquid-air interface, leading to the spontaneous wrapping of the droplet (Py et al., 2007b).

rable with the size of the template, so that the contact line remains pinned at the edges of the plate (the liquid partially wets the plate). Experimentally, as the liquid evaporates, the structure may fold on itself (see Figure 9b) or, conversely, open again if the bending rigidity is too large. Although computing in detail the non-linear coupling between the deformable droplet and the folding plate is complex (Brubaker and Lega, 2016b), theoretical descriptions with simplified (de Langre et al., 2010, Chen et al., 2010) or bidimensional (Brubaker and Lega, 2016b, Péraud and Lauga, 2014) geometries describe almost quantitatively the evolution of the structure as a function of the volume of the droplet. In terms of scaling, the closing condition for a plate of size $L$ is simply set by a balance of the capillary torque with the elastic response $E I / L$ of the plate as a curvature of order $1 / L$ develops. The structure is thus expected to close for $L>L_{c}$ with

$$
L_{c} \sim \ell_{B}
$$

The numerical prefactor depends on the actual shape of the template (Py et al., 2007b). Additional effects such as contact line depinning (Brubaker and Lega, 2016b, Péraud and Lauga, 2014) or gravity can also be taken into account and lead to a large number of competing stable equilibrium states. The equilibrium configurations observed in practice depend on the mode of preparation of the system. An interesting example is when a drop impacts the elastic sheet, triggering capillary folding. Different fold states are observed as a function of the impact velocity (Antkowiak et al., 2011). We believe that the dynamics of elasto-capillary systems, a challenging problem due to non-linear interactions, could become an interesting general subject for future work.

From a more academic point of view, many studies have been dedicated to the case of a partially wetting droplet deposited on a long flexible strip (Rivetti and Neukirch, 2012, Antkowiak et al., 2011), which can be approximated as a two dimensional problem. In this case, the capillary moment is limited by the size of the droplet and scales as $\gamma R w$, where $w$ is the width of the strip. If the strip deflects with an angle $\alpha$, the resulting curvature induced to the strip is of the order of $\alpha / R$ and the elastic restoring moment scales as $E I \alpha / R$. At 
equilibrium, the deflection angle thus reads:

$$
\alpha \sim\left(R / \ell_{B}\right)^{2}
$$

Depending on the boundary conditions and initial conditions, the strip may simply bend as in the case of a droplet sitting on the cantilever of an AFM (Bonaccurso and Butt, 2005), but also undergo snap through instabilities (Fargette et al., 2014) on pre-buckled strips. Finally, if the elastic stiffness varies along the strip, the drop should shift from stiff to soft regions, as it is energetically favorable to minimize the area of the drop in contact with air. This effect would then constitute an analogous for the durotaxis phenomenon observed with bulk materials (Style et al., 2013b).

The more general case of a sessile drop sitting on a flexible disc was considered by several authors in the limit of linear plate theory, which fails as soon as the deflections become comparable with the thickness of the plate (Kern and Müller, 1992, Olives, 1993, Brubaker and Lega, 2016a). In fact, the corresponding axisymmetric bending solutions also involve compressive azimuthal stresses, which leads to buckling and symmetry breaking.

4.1.3. Applications and practical situations. Capillary wrapping is a robust feature, which is observed on various systems systems with different wettability (Geraldi et al., 2013, Bae et al., 2015). At nanoscales, computer simulations even show that graphene sheets are expected to wrap around nanodroplets (Patra et al., 2009). Several practical applications of capillary origami have been envisioned.

Self-folding micro-containers could for instance be used to encapsulate drugs (Fernandes and Gracias, 2012), while three-dimensional photovoltaic cells exhibit enhanced yield (Guo et al., 2009). Elastic self-closing structures are also present in Nature: the flowers of some species of waterlilies are able to close and trap an air bubble when immersed. This phenomenon has recently inspired the development of "elasto-pipettes" which may capture a given volume of liquid from a bath and release it to another one (Reis et al., 2010, Meng et al., 2014).

Once the structure is closed, an additional electric field applied between the droplet and a substrate covered with a dielectric layer may be used to reopen the structure: the charged droplet entrapped in the folded structure is attracted by the ground and the structure tends to unfold as the voltage is increased (Piñeirua et al., 2010). Similarly to classical electrowetting, a characteristic voltage $U_{c}=\sqrt{\gamma d / \epsilon}$ can be defined, where $d$ is the thickness of the dielectric layer and $\epsilon$ its permittivity. This type of actuation was proposed as a way to produce "iridophores" mimicking the color change of particular cells on the skin of cephalopods (Manakasettharn et al., 2011). Magnetic actuation has finally been proposed as a way to manipulate ferrofluid droplets wrapped in a thin plate (Jamin et al., 2011).

4.1.4. Geometrical limit in capillary origami $\left(R \gg \ell_{B}\right)$. In most practical examples presented above, the size of the thin plate is close to the folding threshold, $R \sim \ell_{B}$ so that capillary and elastic forces are of comparable magnitude. Another interesting limit corresponds to the case where capillary forces strongly dominate over bending, $R \gg \ell_{B}$, but are still unable to induce significant stretching. Beautiful experiments were conducted with polystyrene sheets of thickness $t \sim 100 \mathrm{~nm}$ corresponding to $R / \ell_{B}>10^{2}$ and lead to strongly deformed shapes (Figure 10). These equilibrium shapes may be predicted using a geometrical limit where the membrane is unstretchable but can accommodate large compression through the formation of wrinkles (Paulsen et al., 2015). Depending on the amount 

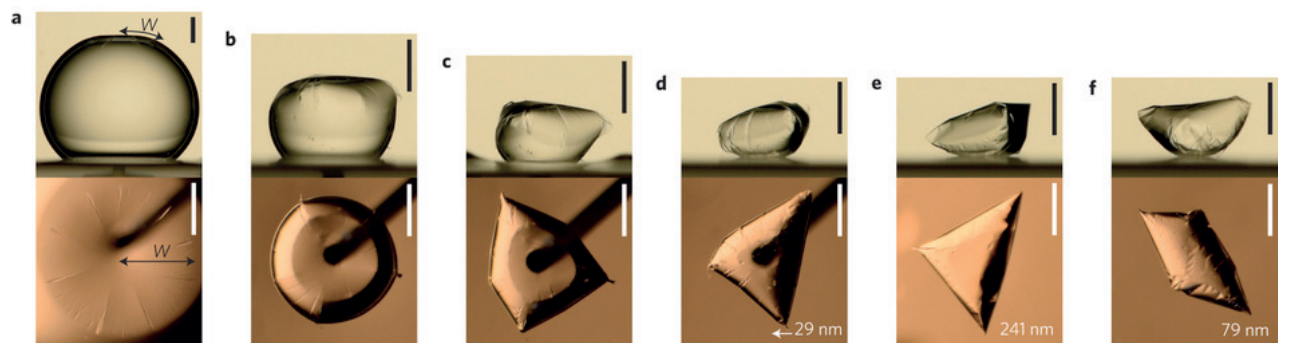

Figure 10

In the limit of very flexible sheets $\left(R \gg \ell_{B}\right)$, capillary wrapping leads to geometrically optimal non-axisymmetric shapes. Scale bar $1 \mathrm{~mm}$ (Paulsen et al., 2015).

of liquid present inside the structure, a circular sheet first adopts a shape analogous to the parachutes described by G.I. Taylor (Taylor, 1963). As the volume of trapped liquid gets small, the global shape undergoes symmetry breaking, leading to structures reminiscent of samosas (Figure 10).

\subsection{Capillary stretching}

The previous situations mostly involve a balance between capillary forces and bending. In this section we examine if surface tension may induce significant stretching in an elastic membrane.

4.2.1. Stretching parameter. Consider a model configuration where a thin disk floats at the surface of a bath of liquid (Figure 11c). We assume here for simplicity that the surface energy of the solid does not depend on strain, so that the notions of surface stress and energy presented in Section 2.1 are here equivalent. The total mechanical tension sustained by the membrane is the surface tension of the liquid $\gamma$, but surface stresses apply along the faces of the membrane, leading to an absolute in-plane strain is $\varepsilon_{1}=\left(\gamma-\gamma_{S V}-\gamma_{S L}\right) / E t$, where $\gamma_{S V}$ and $\gamma_{S L}$ are respectively the solid/vapour and solid/liquid surface energies. This deformation takes as a reference state the solid without interfacial forces, in a putative state where it would be surrounded by the same material (Figure 11a). In practice however, one would measure this deformation with respect to a reference state corresponding to a membrane freely suspended in air (Figure 11b), therefore with an absolute compression induced by surface stress $\varepsilon_{0}=2 \gamma_{S V} / E t$. As a conclusion, depositing the membrane at the surface of the liquid leads to the relative stretching strain:

$$
\varepsilon=\varepsilon_{1}-\varepsilon_{0}=(1+\cos \theta) \mathcal{S} \quad \text { with } \quad \mathcal{S}=\frac{\gamma}{E t}
$$

(29pillary stretching parameter:

where $\theta$ is Young's contact angle. The capillary stretching parameter $\mathcal{S}$ thus corresponds to the characteristic strain induced on a sheet by surface tension or equivalently to the ratio

$$
\mathcal{S}=\frac{\gamma}{E t}
$$
of surface tension to stretching energies.

In general, we therefore expect elasto-capillary systems with a size $L$ to depend on two dimensionless parameters $\left(L / \ell_{B}, \mathcal{S}\right)$. Typical values of $\mathcal{S}$ used in the experiments reported in this review range from $\mathcal{S} \sim 10^{-3}$ to $10^{-6}$ and it would be tempting to disregard stretching effects. Nevertheless, due to boundary conditions or to the geometry of the system, the impact of stretching and in-plane forces may be significant. 

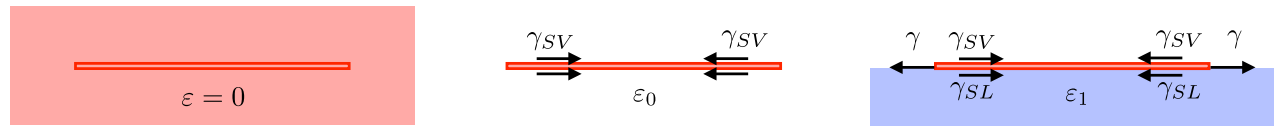

Figure 11

Action of surface stresses on a thin sheet: while the ideal extraction of a membrane would induce a compressive strain $\varepsilon_{0}$, depositing this membrane at the surface of a liquid bath results in a stretching stain $\varepsilon_{1}$. Laying a thin sheet initially sustained in the air thus leads to a relative strain $\varepsilon=\varepsilon_{1}-\varepsilon_{0}$. Nevertheless, the global tension across the membrane is equal to the surface tension of the liquid.

a)

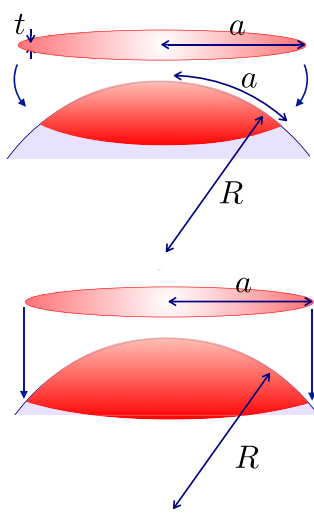

b)

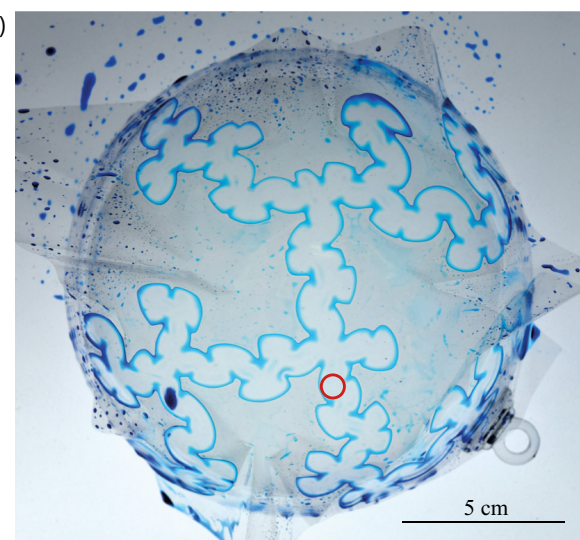

Figure 12

(a) Sticking a thin disk on a sphere faces geometrical incompatibilities: maintaining a constant radius in the disk results in a compression of the perimeter and conversely, maintaining the perimeter constant induces a stretching strain along the radial direction. (b) Depositing a wide adhesive sheet on a sphere leads to complex adhesion patterns, which width (indicated with the red circle) is set by the critical radius $a_{c}$.

4.2.2. Capillary wrapping of a rigid sphere. A first illustration of capillary induced stretching is the case of the wrapping of a solid sphere by a thin sheet coated with a wetting liquid. Consider an ideal case where an initially flat disk of radius $a$ and thickness $t$ perfectly covers a sphere of radius $R$ (Figure 12a). We focus here on the limit where bending is negligible in comparison with capillary adhesion $\left(R \gg \ell_{B}\right)$. Transforming the disk into a spherical cap results in a radial extension and an orthoradial compression of the sheet. Indeed keeping constant the perimeter of the disk as it is embossed to the sphere obviously tends to stretch the radial direction, and conversely, maintaining the diameter results in a excess of perimeter. The scaling for the corresponding strains can be estimated from simple length conservation as $\varepsilon \sim(a / R)^{2}$, which leads to a cost in elastic energy $\mathcal{U}_{e} \sim \operatorname{Eta}^{2}(a / R)^{4}$. Conversely, the gain in adhesion energy simply reads $\mathcal{U}_{s} \sim \gamma a^{2}$. The disk is therefore expected to adhere perfectly on the sphere if its radius is below a critical value (Majidi and Adams, 2009):

$$
a_{c}=R \mathcal{S}^{1 / 4}
$$

Very complex adhesion patterns are experimentally obtained with larger disks (Figure 12b). However, the characteristic width of these patterns remains set by $a_{c}$ (Hure et al., 2011). 
An interesting transition between bending and stretching is also found in the "liquid blister" test (Chopin et al., 2008). A sheet is initially laid on a plate covered with a thin layer of liquid and indented from underneath through a hole in the plate. Different transitions are observed as the displacement $d$ imposed by the indentor is increased. First, the blister remains axisymmetric, and its extension $R$ is dictated by a balance between stretching and adhesion and follows $R \sim d \mathcal{S}^{-1 / 4}$. However due to the increasing stretching energy, the blister eventually takes a triangular shape set by a balance between bending and adhesion.

We see on these examples that capillary adhesion leads to in-plane stresses which are the main players in determining the boundaries of adhesive patches. These stresses are not due to capillary force directly pulling on the boundary of the plate, but are indirectly induced by the distortion in its geometry.

4.2.3. Sheet on a drop. We now describe another scenario for capillary stretching. In an elegant experiment (Figure 13a), a very thin polymeric disk of radius $L$ is laid on a liquid drop of radius $R \gg \ell_{B}$ (King et al., 2012). The combination of capillary traction along the boundary, and Laplace pressure acting on the surface of the sheet tends to impose the radius of curvature of the droplet to the elastic disk, leading, as in the case of the rigid sphere, to radial tension and orthoradial compression. As a consequence, wrinkles appear when the compressive stress induced by the deformation of the disk overcomes the capillary tension, $E t(L / R)^{2} \sim \gamma$, ie $L / R \sim \mathcal{S}^{1 / 2}$. The wavelength of the wrinkles results from a balance between capillary traction and bending stiffness (Paulsen et al., 2016). When the radius of the sphere is decreased (and therefore the compressive orthoradial load increased), localised folds eventually appear and motivate current theoretical developments.

4.2.4. Drop on a floating sheet. Another elastocapillary experiment consists in depositing a tiny drop on a large thin disk floating on bath of liquid (Figure 13b). In the regime of highly compliant sheets (the radius of the drop $R \gg \ell_{B}$ ), an intriguing radial wrinkling pattern is observed (Bodiguel, 2006, Huang et al., 2007, Toga et al., 2013). As in the case described just above, capillary forces produce a dimple in the sheet below the droplet, which results in radial tension. Outside the drop, this tension tends to pull inwards any material circle on the sheet, resulting in azimuthal compression. Wrinkling occurs when compression overcomes the isotropic tension initially induced by capillary traction on the outer boundaries of the floating membrane. While the number $N$ of wrinkles apparently relies on a balance between capillary traction and bending $\left(N \sim \sqrt{R / \ell_{B}}\right)$, the interplay between surface tension and stretching dictates their radial extension $L_{w}$, as experiments were first interpreted to follow $L_{w} \sim R \mathcal{S}^{-1 / 2}$ (Huang et al., 2007). From these two visual observations $\left(N, L_{w}\right)$, one can infer $\ell_{B}$ and $\mathcal{S}$, and therefore estimate nondestructively both the material stiffness $E$ and the thickness of the sheet $t$. This experiment triggered an active research in the last years because the detailed description of the different phenomena is quite subtle. Note for example the period doubling wrinkling close to the contact-line with the drop (Figure 13c), which is reminiscent of the more complete capillary cascade observed in (Huang et al., 2010). Only after several years did theoretical studies obtain a prediction for the extension $L_{w} \sim R \mathcal{S}^{-1 / 3}$, which would also be compatible with experimental data (Schroll et al., 2013).

In an attempt to understand this capillary wrinkling, the simpler situation of an annulus floating on water was first considered: wrinkling appears when the surface tension of the 

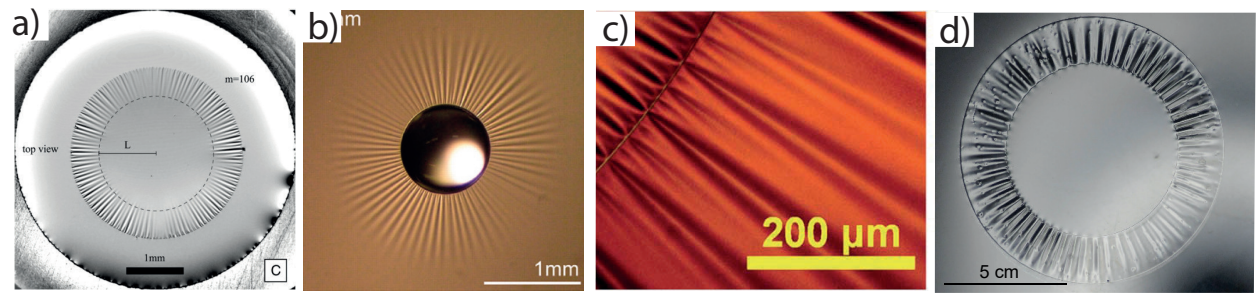

Figure 13

(a) A disk of very flexible membrane wrinkles when laid on a drop (Paulsen et al., 2015). (b) A drop, or a bubble on a very flexible floating membrane generates tension that leads to wrinkling (Huang et al., 2007). (c) Close up of wrinkle patterns close to the contact-line with the drop (Schroll et al., 2013). (d) Wrinkles are also observed on a floating annulus subject to differential surface tension (Piñeirua et al., 2013).

outer liquid $\gamma_{o}$ is significantly lower than that of the inner liquid surface $\gamma_{i}$ (for instance if surfactant is added outside the annulus). Indeed, the stronger inner tension $\gamma_{i}$ tends to reduce the radius of the inner hole, leading to azimuthal compression of the annulus along its inner boundary, and eventually to buckling. A first experimental realisation of this problem in a macroscopic system (Piñeirua et al., 2013) was limited to a different regime, where the buckling wavelength was determined by the interplay of bending rigidity and underlying liquid weight (Figure 13d). However in the limit of infinitely flexible sheets, or $R / \ell_{B} \ll 1$, theory shows that wrinkles extend over a distance $L_{w}=R \gamma_{i} / 2 \gamma_{o}$, if $R$ is the radius of the inner hole in an infinite membrane (Davidovitch et al., 2011). When returning to the initial problem of the drop on a sheet, this result was used with the drop-induced pulling stresses $\gamma_{i}$ which was estimated as $\gamma_{i}^{3} \sim E t \gamma_{o}^{2}$ in the limit of infinitely flexible sheets, which led to $L_{w} \sim$ $R\left(E t / \gamma_{0}\right)^{1 / 3} \sim R S^{-1 / 3}$. However, the richness of the simplified problem is illustrated by recent experiments using ultra-thin sheets, which showed that far above buckling threshold, a partial collapse of a finite annulus may also occur (Paulsen et al., 2017).

If the liquid drop is now deposited on a very thin elastomer membrane clamped along its remote boundary, the drop strongly deforms the membrane (Schulman and Dalnoki-Veress, 2015) seen as an extremely compliant substrate. From the observation of the apparent contact angles, the tension in the membrane may be measured, which may allow to infer the surface stress of the elastomer (Nadermann et al., 2013). Droplets deposited on opposite sides of the membranes interact through the out-of-plane deformation of the membrane that they induce, and therefore spontaneously assemble (Liu et al., 2017).

\section{CONCLUSION}

Although surface tension is usually not considered in fluid-structure interactions involving large scales, capillary forces become dominant at submillimetric scale and may deform micro-objects. We focused in this review on three main types of deformation occurring at different dimensions. 3D deformations induced by capillarity to an elastic solid occur on a "shear" elastocapillary length $\ell_{S} \sim \Upsilon / G$. The parameter $\Upsilon$ represents the surface stress acting at the surface of a solid in the case of an object immersed in a fluid. It can also be taken as the surface tension of a liquid $\gamma$ when the deformations induced by a 
sessile droplet at the surface of a solid are considered. Since this length scale is atomic for usual engineering materials, elastocapillary phenomena have long been neglected. However, the growing development of soft polymers gives access to materials with Young's moduli 5 to 10 orders of magnitude smaller than steel, paving the way to stimulating studies on elasto-capillarity at the macroscopic scale.

Bending, buckling, bundling or coiling deformations in 1D slender structures give rise to spectacular shapes and patterns. The typical curvature induced by surface tension forces may be described by the bending length $\ell_{B} \sim \sqrt{E t^{3} / \gamma}$, where $E t^{3}$ is the bending modulus for a structure of thickness $t$.

The bending of slender structures may be extended to $2 \mathrm{D}$ sheets. However, geometrical constraints result into stretching and compression, which may lead to complex buckling instabilities and beautiful patterns such as wrinkles or folds. The effect of stretching/compression is characterized by a stretching parameter $\mathcal{S}=\gamma / E t$, which can also be viewed as the comparison of $\ell_{S}$ with the thickness of the structure.

As a conclusion, elasto-capillarity thus describes fascinating phenomena across many length scales. It covers a wide range of academic and applied problems from static and dynamic wetting and adhesion to the self-assembly of slender structures or the generation of 3D shapes from flat templates. More particularly, the field of biophysics driven by the better control of mechanics at the cellular level, is certainly mature for numerous upcoming applications. Campàs et al. for instance nicely demonstrate how capillary pressure may be used for in vivo probing of living cells (Campàs et al., 2013). Tubes of biological membrane are also known to undergo an instability reminiscent of elasto-capillary instability of soft gels, which leads to the fragmentation of mitochondria through pearling (Gonzalez-Rodriguez et al., 2015). The development of micro- and nano-engineered mechanical devices is also likely to raise new elasto-capillary problems.

\section{SUMMARY POINTS}

1. The 3D deformation of a bulk solid induced by surface tension occur at the "shear" length scale $\ell_{S}=\Upsilon / G$. This scale is atomic for traditional engineering materials but may become macroscopic when soft polymeric or biological materials are involved.

2. Depositing a liquid droplet at the surface of a soft elastic solid induces the formation of a wetting ridge of typical extension $\ell_{S}$, which affects the static and dynamic wetting properties of the liquid.

3. Capillary induced moments may bend 1D slender structures. The corresponding curvature is dictated by a "bending" length $\ell_{B} \sim \sqrt{E t^{3} / \gamma}$.

4. This bending effect may be extended to $2 \mathrm{D}$ sheets or shells although geometrical constrains may induce the stretching or compression of the structure. This additional effect is characterised by a "stretching" number $\mathcal{S}=\gamma / E t$.

\section{FUTURE ISSUES}

1. Depositing a liquid droplet on a soft substrate induces the formation of a ridge of typical size $\ell_{S}$. However, the physical cut-offs of the stress singularities at the contact line remain to be confirmed. Most soft materials are composed of polymers 
or gels and the evolution of surface stresses with strain is not obvious.

Determining experimentally such stresses would also be important since a strong variation of $\Upsilon$ in the vicinity of the ridge should modify its profile.

More generally, understanding the dynamics of the deformation of soft solids would be crucial. Measuring the dynamics of droplet sliding on a soft substrate may indeed constitute a rheometry technique to probe soft materials.

2. Two different mechanisms seem to explain the formation of bundles obtained experimentally with arrays of wet fibres. However the limit of validity of these scenarios remains to be confirmed. More generally, understanding the bundling dynamics would be very valuable. Several works have been dedicated to flexible rods, but the general case of $2 \mathrm{D}$ arrays of fibres remains an open question.

3. Although large amplitude deformation of thin sheets mainly rely on bending, stretching and compression may also play an important role due to geometrical incompatibilities. A consequence of this additional ingredient is the formation of wrinkles, folds or crumples, especially in the case of very thin sheets or shells (of size $L \gg \ell_{B}$ ). The description of such complex structures would deserve a comprehensive review.

Beyond static shapes, understanding the wrapping dynamics would also be important from a practical point of view since the final structure may rely on the order of folding events.

4. Finally, building up on the framework of elastocapillary phenomena may pave the way to explore other surface interactions such as adhesion and even fracture, which can also be described in terms of surface energy. Nevertheless additional effects such as friction may have to be added.

\section{DISCLOSURE STATEMENT}

The authors are not aware of any affiliations, memberships, funding, or financial holdings that might be perceived as affecting the objectivity of this review.

\section{ACKNOWLEDGMENTS}

We thank tienne Guyon for the useful comments on our manuscript.

This work was partly funded by the Interuniversity Attraction Poles Programme (IAP 7/38

MicroMAST) initiated by the Belgian Science Policy Office and the Fonds National de la Recherche Scientifique.

\section{LITERATURE CITED}

Abe and Reed, 1996.Abe, T. and Reed, M. L. (1996). Control of liquid bridging induced stiction of micromechanical structures. Journal of Micromechanics and Microengineering, 6(2):213-217.

Adami and Caps, 2015.Adami, N. and Caps, H. (2015). Surface tension profiles in vertical soap films. Physical Review E - Statistical, Nonlinear, and Soft Matter Physics, 91(1):1-7.

Alizadeh et al., 2013.Alizadeh, A., Bahadur, V., Shang, W., Zhu, Y., Buckley, D., Dhinojwala, A., and Sohal, M. (2013). Influence of Substrate Elasticity on Droplet Impact Dynamics. Langmuir, 29(14):4520-4524. 
Andreotti et al., 2016.Andreotti, B., Bäumchen, O., Boulogne, F., Daniels, K. E., Dufresne, E. R., Perrin, H., Salez, T., Snoeijer, J. H., and Style, R. W. (2016). Solid capillarity: when and how does surface tension deform soft solids? Soft Matter, 12(12):2993-2996.

Andreotti et al., 2011.Andreotti, B., Marchand, A., Das, S., and Snoeijer, J. H. (2011). Elastocapillary instability under partial wetting conditions: Bending versus buckling. Physical Review E, 84(6):061601.

Andreotti and Snoeijer, 2016.Andreotti, B. and Snoeijer, J. H. (2016). Soft wetting and the Shuttleworth effect, at the crossroads between thermodynamics and mechanics. EPL (Europhysics Letters), 113(6):66001.

Anoop and Sen, 2015.Anoop, R. and Sen, A. K. (2015). Capillary flow enhancement in rectangular polymer microchannels with a deformable wall. Physical Review E, 92(1):013024.

Antkowiak et al., 2011.Antkowiak, A., Audoly, B., Josserand, C., Neukirch, S., and Rivetti, M. (2011). Instant fabrication and selection of folded structures using drop impact. Proceedings of the National Academy of Sciences, 108(26):10400-10404.

Aristoff et al., 2011.Aristoff, J. M., Duprat, C., and Stone, H. A. (2011). Elastocapillary imbibition. International Journal of Non-Linear Mechanics, 46(4):648-656.

Audoly and Pomeau, 2010.Audoly, B. and Pomeau, Y. (2010). Elasticity and Geometry: From hair curls to the non-linear response of shells. Oxford University Press, Oxford.

Bae et al., 2015.Bae, J., Ouchi, T., and Hayward, R. C. (2015). Measuring the Elastic Modulus of Thin Polymer Sheets by Elastocapillary Bending. ACS Applied Materials \& Interfaces, 7(27):14734-14742.

Barrière et al., 1996.Barrière, B., Sekimoto, K., and Leibler, L. (1996). Peristaltic instability of cylindrical gels. The Journal of Chemical Physics, 105(4):1735-1738.

Bernardino and Dietrich, 2012.Bernardino, N. R. and Dietrich, S. (2012). Complete wetting of elastically responsive substrates. Physical Review E, 85(5):051603.

Bico et al., 2004.Bico, J., Roman, B., Moulin, L., and Boudaoud, A. (2004). Adhesion: Elastocapillary coalescence in wet hair. Nature, 432(7018):690-690.

Bodiguel, 2006.Bodiguel, H. (2006). Propriétés mécaniques de films polymère ultraminces. PhD thesis, Paris VI.

Bonaccurso and Butt, 2005.Bonaccurso, E. and Butt, H.-J. (2005). Microdrops on Atomic Force Microscope Cantilevers: Evaporation of Water and Spring Constant Calibration. The Journal of Physical Chemistry B, 109(1):253-263.

Boncheva and Whitesides, 2005.Boncheva, M. and Whitesides, G. M. (2005). Making Things by Self-Assembly. MRS Bulletin, 30(10):736-742.

Boudaoud et al., 2007.Boudaoud, A., Bico, J., and Roman, B. (2007). Elastocapillary coalescence: Aggregation and fragmentation with a maximal size. Physical Review E - Statistical, Nonlinear, and Soft Matter Physics, 76(6):1-4.

Brubaker and Lega, 2016a.Brubaker, N. D. and Lega, J. (2016a). Capillary-induced deformations of a thin elastic sheet. Philosophical Transactions of the Royal Society A: Mathematical, Physical and Engineering Sciences, 374(2066):20150169.

Brubaker and Lega, 2016b.Brubaker, N. D. and Lega, J. (2016b). Two-dimensional capillary origami. Physics Letters A, 380(1-2):83-87.

Cambau et al., 2011.Cambau, T., Bico, J., and Reyssat, E. (2011). Capillary rise between flexible walls. EPL (Europhysics Letters), 96(2):24001.

Campàs et al., 2013.Campàs, O., Mammoto, T., Hasso, S., Sperling, R. A., O'Connell, D., Bischof, A. G., Maas, R., Weitz, D. A., Mahadevan, L., and Ingber, D. E. (2013). Quantifying cellgenerated mechanical forces within living embryonic tissues. Nature Methods, 11(2):183-189.

Carré et al., 1996.Carré, A., Gastel, J.-C., and Shanahan, M. E. R. (1996). Viscoelastic effects in the spreading of liquids. Nature, 379(6564):432-434.

Chakrapani et al., 2004.Chakrapani, N., Wei, B., Carrillo, A., Ajayan, P. M., and Kane, R. S. (2004). Capillarity-driven assembly of two-dimensional cellular carbon nanotube foams. Pro- 
ceedings of the National Academy of Sciences, 101(12):4009-4012.

Chandra and Yang, 2009.Chandra, D. and Yang, S. (2009). Capillary-Force-Induced Clustering of Micropillar Arrays: Is It Caused by Isolated Capillary Bridges or by the Lateral Capillary Meniscus Interaction Force? Langmuir, 25(18):10430-10434.

Chandra and Yang, 2010.Chandra, D. and Yang, S. (2010). Stability of High-Aspect-Ratio Micropillar Arrays against Adhesive and Capillary Forces. Accounts of Chemical Research, 43(8):10801091.

Chaudhury et al., 1996.Chaudhury, M. K., Weaver, T., Hui, C. Y., and Kramer, E. J. (1996). Adhesive contact of cylindrical lens and a flat sheet. Journal of Applied Physics, 80(1):30-37.

Chen et al., 2012.Chen, D., Cai, S., Suo, Z., and Hayward, R. C. (2012). Surface Energy as a Barrier to Creasing of Elastomer Films: An Elastic Analogy to Classical Nucleation. Physical Review Letters, 109(3):038001.

Chen et al., 2010.Chen, L., Wang, X., Wen, W., and Li, Z. (2010). Critical droplet volume for spontaneous capillary wrapping. Applied Physics Letters, 97(12):124103.

Chiodi et al., 2010.Chiodi, F., Roman, B., and Bico, J. (2010). Piercing an interface with a brush: Collaborative stiffening. EPL (Europhysics Letters), 90(4):44006.

Cho et al., 2010.Cho, J.-h., Azam, A., and Gracias, D. H. (2010). Three Dimensional Nanofabrication Using Surface Forces . Langmuir, 26(21):16534-16539.

Chopin et al., 2008.Chopin, J., Vella, D., and Boudaoud, A. (2008). The liquid blister test. Proceedings of the Royal Society A: Mathematical, Physical and Engineering Sciences, 464(2099):28872906.

Cohen and Mahadevan, 2003.Cohen, A. E. and Mahadevan, L. (2003). Kinks, rings, and rackets in filamentous structures. Proceedings of the National Academy of Sciences of the United States of America, 100(21):12141-6.

Correa-Duarte et al., 2004.Correa-Duarte, M. A., Wagner, N., Rojas-Chapana, J., Morsczeck, C., Thie, M., and Giersig, M. (2004). Fabrication and Biocompatibility of Carbon Nanotube-Based 3D Networks as Scaffolds for Cell Seeding and Growth. Nano Letters, 4(11):2233-2236.

Crane et al., 2013.Crane, N. B., Onen, O., Carballo, J., Ni, Q., and Guldiken, R. (2013). Fluidic assembly at the microscale: progress and prospects. Microfluidics and Nanofluidics, 14(3-4):383419.

Cuenot et al., 2004.Cuenot, S., Frétigny, C., Demoustier-Champagne, S., and Nysten, B. (2004). Surface tension effect on the mechanical properties of nanomaterials measured by atomic force microscopy. Physical Review B, 69(16):165410.

Das et al., 2011.Das, S., Marchand, A., Andreotti, B., and Snoeijer, J. H. (2011). Elastic deformation due to tangential capillary forces. Physics of Fluids, 23(7):072006.

Davidovitch et al., 2011.Davidovitch, B., Schroll, R. D., Vella, D., Adda-Bedia, M., and Cerda, E. A. (2011). Prototypical model for tensional wrinkling in thin sheets. Proceedings of the National Academy of Sciences, 108(45):18227-18232.

de Gennes et al., 2004.de Gennes, P.-G., Brochard-Wyart, F., and Quéré, D. (2004). Capillarity and Wetting Phenomena. Springer New York, New York, NY.

de Langre et al., 2010.de Langre, E., Baroud, C., and Reverdy, P. (2010). Energy criteria for elastocapillary wrapping. Journal of Fluids and Structures, 26(2):205-217.

De Volder and Hart, 2013.De Volder, M. and Hart, A. J. (2013). Engineering Hierarchical Nanostructures by Elastocapillary Self-Assembly. Angewandte Chemie International Edition, $52(9): 2412-2425$.

De Volder et al., 2010.De Volder, M., Tawfick, S. H., Park, S. J., Copic, D., Zhao, Z., Lu, W., and Hart, A. J. (2010). Diverse 3D Microarchitectures Made by Capillary Forming of Carbon Nanotubes. Advanced Materials, 22(39):4384-4389.

De Volder et al., 2011.De Volder, M. F. L., Tawfick, S., Park, S. J., and Hart, A. J. (2011). Corrugated Carbon Nanotube Microstructures with Geometrically Tunable Compliance. ACS Nano, $5(9): 7310-7317$. 
DelRio et al., 2005.DelRio, F. W., de Boer, M. P., Knapp, J. A., David Reedy, E., Clews, P. J., and Dunn, M. L. (2005). The role of van der Waals forces in adhesion of micromachined surfaces. Nature Materials, 4(8):629-634.

Duan and Berggren, 2010.Duan, H. and Berggren, K. K. (2010). Directed Self-Assembly at the 10 $\mathrm{nm}$ Scale by Using Capillary Force-Induced Nanocohesion. Nano Letters, 10(9):3710-3716.

Ducloué et al., 2014.Ducloué, L., Pitois, O., Goyon, J., Chateau, X., and Ovarlez, G. (2014). Coupling of elasticity to capillarity in soft aerated materials. Soft Matter, 10(28):5093.

Duprat et al., 2011.Duprat, C., Aristoff, J. M., and Stone, H. A. (2011). Dynamics of elastocapillary rise. Journal of Fluid Mechanics, 679(2011):641-654.

Duprat and Protiere, 2015.Duprat, C. and Protiere, S. (2015). Capillary stretching of fibers. EPL (Europhysics Letters), 111(5):56006.

Duprat et al., 2012.Duprat, C., Protière, S., Beebe, a. Y., and Stone, H. a. (2012). Wetting of flexible fibre arrays. Nature, 482(7386):510-3.

Duprat and Stone, 2015.Duprat, C. and Stone, H., editors (2015). Fluid-Structure Interactions in Low-Reynolds-Number Flows. RSC Soft Matter Series. Royal Society of Chemistry, Cambridge.

Elettro et al., 2016.Elettro, H., Neukirch, S., Vollrath, F., and Antkowiak, A. (2016). In-drop capillary spooling of spider capture thread inspires hybrid fibers with mixed solidliquid mechanical properties. Proceedings of the National Academy of Sciences, 113(22):6143-6147.

Eshelby, 1957.Eshelby, J. D. (1957). The Determination of the Elastic Field of an Ellipsoidal Inclusion, and Related Problems. Proceedings of the Royal Society of London. Series A, Mathematical and Physical Sciences, 241(1226):376-396.

Extrand and Kumagai, 1996.Extrand, C. and Kumagai, Y. (1996). Contact Angles and Hysteresis on Soft Surfaces. Journal of Colloid and Interface Science, 184(1):191-200.

Fargette et al., 2014.Fargette, A., Neukirch, S., and Antkowiak, A. (2014). Elastocapillary Snapping: Capillarity Induces Snap-Through Instabilities in Small Elastic Beams. Physical Review Letters, 112(13):137802.

Fernandes and Gracias, 2012.Fernandes, R. and Gracias, D. H. (2012). Self-folding polymeric containers for encapsulation and delivery of drugs . Advanced Drug Delivery Reviews, 64(14):15791589.

Filipiak et al., 2009.Filipiak, D. J., Azam, A., Leong, T. G., and Gracias, D. H. (2009). Hierarchical self-assembly of complex polyhedral microcontainers. Journal of Micromechanics and Microengineering, 19(7):075012.

García et al., 2007.García, E. J., Hart, A. J., Wardle, B. L., and Slocum, A. H. (2007). Fabrication of composite microstructures by capillarity-driven wetting of aligned carbon nanotubes with polymers. Nanotechnology, 18(16):165602.

Geraldi et al., 2013.Geraldi, N. R., Ouali, F. F., Morris, R. H., McHale, G., and Newton, M. I. (2013). Capillary origami and superhydrophobic membrane surfaces. Applied Physics Letters, 102(21):214104.

Gernay et al., 2016.Gernay, S., Federle, W., Lambert, P., and Gilet, T. (2016). Elasto-capillarity in insect fibrillar adhesion. Journal of The Royal Society Interface, 13(121).

Gonzalez-Rodriguez et al., 2015.Gonzalez-Rodriguez, D., Sart, S., Babataheri, A., Tareste, D., Barakat, A. I., Clanet, C., and Husson, J. (2015). Elastocapillary Instability in Mitochondrial Fission. Physical Review Letters, 115(8):088102.

Grotberg and Jensen, 2004.Grotberg, J. B. and Jensen, O. E. (2004). BIOFLUID MECHANICS IN FLEXIBLE TUBES. Annual Review of Fluid Mechanics, 36(1):121-147.

Guo et al., 2009.Guo, X., Li, H., Yeop Ahn, B., Duoss, E. B., Hsia, K. J., Lewis, J. A., and Nuzzo, R. G. (2009). Two- and three-dimensional folding of thin film single-crystalline silicon for photovoltaic power applications. Proceedings of the National Academy of Sciences, 106(48):2014920154.

Hadjittofis et al., 2016.Hadjittofis, A., Lister, J. R., Singh, K., and Vella, D. (2016). Evaporation effects in elastocapillary aggregation. Journal of Fluid Mechanics, 792:168-185. 
Hazel and Heil, 2003.Hazel, A. L. and Heil, M. (2003). Three-dimensional airway reopening: the steady propagation of a semi-infinite bubble into a buckled elastic tube. Journal of Fluid Mechanics, 478:47-70.

Henann and Bertoldi, 2014.Henann, D. L. and Bertoldi, K. (2014). Modeling of elasto-capillary phenomena. Soft Matter, 10(5):709-717.

Howland et al., 2016.Howland, C. J., Antkowiak, A., Castrejón-Pita, J. R., Howison, S. D., Oliver, J. M., Style, R. W., and Castrejón-Pita, A. A. (2016). It's Harder to Splash on Soft Solids. Physical Review Letters, 117(18):184502.

Hu et al., 2010.Hu, M., Ou, F. S., Wu, W., Naumov, I., Li, X., Bratkovsky, A. M., Williams, R. S., and Li, Z. (2010). Gold Nanofingers for Molecule Trapping and Detection. Journal of the American Chemical Society, 132(37):12820-12822.

Hu et al., 2015.Hu, Y., Lao, Z., Cumming, B. P., Wu, D., Li, J., Liang, H., Chu, J., Huang, W., and $\mathrm{Gu}, \mathrm{M}$. (2015). Laser printing hierarchical structures with the aid of controlled capillary-driven self-assembly. Proceedings of the National Academy of Sciences, 112(22):6876-6881.

Hu et al., 2003.Hu, Y., Lee, K. Y. C., and Israelachvili, J. (2003). Sealed Minitrough for Microscopy and Long-Term Stability Studies of Langmuir Monolayers. Langmuir, 19(1):100-104.

Huang et al., 2010.Huang, J., Davidovitch, B., Santangelo, C. D., Russell, T. P., and Menon, N. (2010). Smooth Cascade of Wrinkles at the Edge of a Floating Elastic Film. Physical Review Letters, 105(3):038302.

Huang et al., 2007.Huang, J., Juszkiewicz, M., de Jeu, W. H., Cerda, E., Emrick, T., Menon, N., and Russell, T. P. (2007). Capillary Wrinkling of Floating Thin Polymer Films. Science, 317(5838):650-653.

Hui et al., 2002.Hui, C. Y., Jagota, A., Lin, Y. Y., and Kramer, E. J. (2002). Constraints on Microcontact Printing Imposed by Stamp Deformation. Langmuir, 18(4):1394-1407.

Hure et al., 2011.Hure, J., Roman, B., and Bico, J. (2011). Wrapping an Adhesive Sphere with an Elastic Sheet. Physical Review Letters, 106(17):174301.

Jagota et al., 2012.Jagota, A., Paretkar, D., and Ghatak, A. (2012). Surface-tension-induced flattening of a nearly plane elastic solid. Physical Review E, 85(5):051602.

Jamin et al., 2011.Jamin, T., Py, C., and Falcon, E. (2011). Instability of the Origami of a Ferrofluid Drop in a Magnetic Field. Physical Review Letters, 107(20):204503.

Jerison et al., 2011.Jerison, E. R., Xu, Y., Wilen, L. A., and Dufresne, E. R. (2011). Deformation of an Elastic Substrate by a Three-Phase Contact Line. Physical Review Letters, 106(18):186103.

Kajiya et al., 2013.Kajiya, T., Daerr, A., Narita, T., Royon, L., Lequeux, F., and Limat, L. (2013). Advancing liquid contact line on visco-elastic gel substrates: stick-slip vs. continuous motions. Soft Matter, 9(2):454-461.

Kang et al., 2010.Kang, S. H., Pokroy, B., Mahadevan, L., and Aizenberg, J. (2010). Control of Shape and Size of Nanopillar Assembly by Adhesion-Mediated Elastocapillary Interaction. ACS Nano, 4(11):6323-6331.

Karpitschka et al., 2015.Karpitschka, S., Das, S., van Gorcum, M., Perrin, H., Andreotti, B., and Snoeijer, J. H. (2015). Droplets move over viscoelastic substrates by surfing a ridge. Nature Communications, 6:7891.

Kern and Müller, 1992.Kern, R. and Müller, P. (1992). Deformation of an elastic thin solid induced by a liquid droplet. Surface Science, 264(3):467-494.

Kim and Mahadevan, 2006.Kim, H.-Y. and Mahadevan, L. (2006). Capillary rise between elastic sheets. Journal of Fluid Mechanics, 548(-1):141.

Kim et al., 2016.Kim, T.-H., Kim, J., and Kim, H.-y. (2016). Evaporation-driven clustering of microscale pillars and lamellae. Physics of Fluids, 28(2):022003.

King et al., 2012.King, H., Schroll, R. D., Davidovitch, B., and Menon, N. (2012). Elastic sheet on a liquid drop reveals wrinkling and crumpling as distinct symmetry-breaking instabilities. Proceedings of the National Academy of Sciences, 109(25):9716-9720.

Kralchevsky and Nagayama, 2000.Kralchevsky, P. A. and Nagayama, K. (2000). Capillary interac- 
tions between particles bound to interfaces, liquid films and biomembranes. Advances in Colloid and Interface Science, 85(2-3):145-192.

Lau et al., 2003.Lau, K. K. S., Bico, J., Teo, K. B. K., Chhowalla, M., Amaratunga, G. A. J., Milne, W. I., McKinley, G. H., and Gleason, K. K. (2003). Superhydrophobic Carbon Nanotube Forests. Nano Letters, 3(12):1701-1705.

Legrain et al., 2016.Legrain, A., Berenschot, E. J. W., Abelmann, L., Bico, J., and Tas, N. R. (2016). Let's twist again: elasto-capillary assembly of parallel ribbons. Soft Matter, 12(34):71867194.

Legrain et al., 2014.Legrain, A., Janson, T. G., Berenschot, J. W., Abelmann, L., and Tas, N. R. (2014). Controllable elastocapillary folding of three-dimensional micro-objects by through-wafer filling. Journal of Applied Physics, 115(21):214905.

Leong et al., 2010.Leong, T. G., Zarafshar, A. M., and Gracias, D. H. (2010). Three-Dimensional Fabrication at Small Size Scales. Small, 6(7):792-806.

Lester, 1961.Lester, G. (1961). Contact angles of liquids at deformable solid surfaces. Journal of Colloid Science, 16(4):315-326.

Liang et al., 2016.Liang, H., Cao, Z., and Dobrynin, A. V. (2016). Molecular Dynamics Simulations of the Effect of Elastocapillarity on Reinforcement of Soft Polymeric Materials by Liquid Inclusions. Macromolecules, 49(18):7108-7115.

Limat, 2012.Limat, L. (2012). Straight contact lines on a soft, incompressible solid. The European Physical Journal E, 35(12):134.

Liu et al., 2017.Liu, T., Xu, X., Nadermann, N., He, Z., Jagota, A., and Hui, C.-Y. (2017). Interaction of Droplets Separated by an Elastic Film. Langmuir, 33(1):75-81.

Long et al., 1996.Long, D., Ajdari, A., and Leibler, L. (1996). Static and Dynamic Wetting Properties of Thin Rubber Films. Langmuir, 12(21):5221-5230.

M. M. Nicolson, 1955.M. M. Nicolson (1955). Surface Tension in Ionic Crystals. Proceedings of the Royal Society of London. Series A, Mathematical and Physical Sciences, 228(1175):490-510.

Maboudian, 1997.Maboudian, R. (1997). Critical Review: Adhesion in surface micromechanical structures. Journal of Vacuum Science \& Technology B: Microelectronics and Nanometer Structures, 15(1):1.

Majidi and Adams, 2009.Majidi, C. and Adams, G. G. (2009). A simplified formulation of adhesion problems with elastic plates. Proceedings of the Royal Society A: Mathematical, Physical and Engineering Sciences, 465(2107):2217-2230.

Manakasettharn et al., 2011.Manakasettharn, S., Ashley Taylor, J., and Krupenkin, T. N. (2011). Bio-inspired artificial iridophores based on capillary origami: Fabrication and device characterization. Applied Physics Letters, 99(14):144102.

Marchand et al., 2012.Marchand, A., Das, S., Snoeijer, J. H., and Andreotti, B. (2012). Contact Angles on a Soft Solid: From Young's Law to Neumann's Law. Physical Review Letters, 109(23):236101.

Martel et al., 1999.Martel, R., Shea, H. R., and Avouris, P. (1999). Ring Formation in Single-Wall Carbon Nanotubes. The Journal of Physical Chemistry B, 103(36):7551-7556.

Mastrangeli et al., 2009.Mastrangeli, M., Abbasi, S., Varel, C., Van Hoof, C., Celis, J.-P., and Böhringer, K. F. (2009). Self-assembly from milli- to nanoscales: methods and applications. Journal of Micromechanics and Microengineering, 19(8):083001.

Matsuo and Tanaka, 1992.Matsuo, E. S. and Tanaka, T. (1992). Patterns in shrinking gels. Nature, 358(6386):482-485.

Mehrabian et al., 2016.Mehrabian, H., Harting, J., and Snoeijer, J. H. (2016). Soft particles at a fluid interface. Soft Matter, 12(4):1062-1073.

Meng et al., 2014.Meng, Q., Wang, Q., Liu, H., and Jiang, L. (2014). A bio-inspired flexible fiber array with an open radial geometry for highly efficient liquid transfer. NPG Asia Materials, 6(9):e125.

Mora et al., 2011.Mora, S., Abkarian, M., Tabuteau, H., and Pomeau, Y. (2011). Surface instability 
of soft solids under strain. Soft Matter, 7(22):10612.

Mora et al., 2013.Mora, S., Maurini, C., Phou, T., Fromental, J.-m., Audoly, B., and Pomeau, Y. (2013). Solid Drops: Large Capillary Deformations of Immersed Elastic Rods. Physical Review Letters, 111(11):114301.

Mora et al., 2010.Mora, S., Phou, T., Fromental, J.-m., Pismen, L. M., and Pomeau, Y. (2010). Capillarity Driven Instability of a Soft Solid. Physical Review Letters, 105(21):214301.

Mora and Pomeau, 2015.Mora, S. and Pomeau, Y. (2015). Softening of edges of solids by surface tension. Journal of Physics: Condensed Matter, 27(19):194112.

Nadermann et al., 2013.Nadermann, N., Hui, C.-Y., and Jagota, A. (2013). Solid surface tension measured by a liquid drop under a solid film. Proceedings of the National Academy of Sciences, 110(26):10541-10545.

Neukirch et al., 2007.Neukirch, S., Roman, B., de Gaudemaris, B., and Bico, J. (2007). Piercing a liquid surface with an elastic rod: Buckling under capillary forces. Journal of the Mechanics and Physics of Solids, 55(6):1212-1235.

Olives, 1993. Olives, J. (1993). Capillarity and elasticity. The example of the thin plate. Journal of Physics: Condensed Matter, 5(14):2081-2094.

Paretkar et al., 2014.Paretkar, D., Xu, X., Hui, C.-Y., and Jagota, A. (2014). Flattening of a patterned compliant solid by surface stress. Soft Matter, 10(23):4084.

Park et al., 2014.Park, S. J., Schmidt, A. J., Tawfick, S. H., and Hart, A. J. (2014). Precise control of elastocapillary densification of nanostructures via low-pressure condensation. Journal of Micromechanics and Microengineering, 24(6):065019.

Patra et al., 2009.Patra, N., Wang, B., and Kral, P. (2009). Nanodroplet Activated and Guided Folding of Graphene Nanostructures. Nano Letters, 9(11):3766-3771.

Paulsen et al., 2015.Paulsen, J. D., Démery, V., Santangelo, C. D., Russell, T. P., Davidovitch, B., and Menon, N. (2015). Optimal wrapping of liquid droplets with ultrathin sheets. Nature Materials, 14(12):1206-1209.

Paulsen et al., 2017.Paulsen, J. D., Démery, V., Toga, K. B., Qiu, Z., Russell, T. P., Davidovitch, B., and Menon, N. (2017). Geometry-Driven Folding of a Floating Annular Sheet. Physical Review Letters, 118(4):048004.

Paulsen et al., 2016.Paulsen, J. D., Hohlfeld, E., King, H., Huang, J., Qiu, Z., Russell, T. P., Menon, N., Vella, D., and Davidovitch, B. (2016). Curvature-induced stiffness and the spatial variation of wavelength in wrinkled sheets. Proceedings of the National Academy of Sciences, 113(5):1144-1149.

Péraud and Lauga, 2014.Péraud, J.-P. and Lauga, E. (2014). Geometry and wetting of capillary folding. Physical Review E, 89(4):043011.

Pericet-Camara et al., 2008.Pericet-Camara, R., Best, A., Butt, H.-J., and Bonaccurso, E. (2008). Effect of Capillary Pressure and Surface Tension on the Deformation of Elastic Surfaces by Sessile Liquid Microdrops: An Experimental Investigation. Langmuir, 24(19):10565-10568.

Pham et al., 2013.Pham, J. T., Lawrence, J., Lee, D. Y., Grason, G. M., Emrick, T., and Crosby, A. J. (2013). Highly Stretchable Nanoparticle Helices Through Geometric Asymmetry and Surface Forces. Advanced Materials, 25(46):6703-6708.

Piñeirua et al., 2010.Piñeirua, M., Bico, J., and Roman, B. (2010). Capillary origami controlled by an electric field. Soft Matter, 6(18):4491.

Piñeirua et al., 2013.Piñeirua, M., Tanaka, N., Roman, B., and Bico, J. (2013). Capillary buckling of a floating annulus. Soft Matter, 9(46):10985.

Pokroy et al., 2009.Pokroy, B., Kang, S. H., Mahadevan, L., and Aizenberg, J. (2009). SelfOrganization of a Mesoscale Bristle into Ordered, Hierarchical Helical Assemblies. Science, $323(5911): 237-240$.

Py et al., 2007a.Py, C., Bastien, R., Bico, J., Roman, B., and Boudaoud, A. (2007a). 3D aggregation of wet fibers. Europhysics Letters (EPL), 77(4):44005.

Py et al., 2007b.Py, C., Reverdy, P., Doppler, L., Bico, J., Roman, B., and Baroud, C. N. (2007b). 
Capillary Origami: Spontaneous Wrapping of a Droplet with an Elastic Sheet. Physical Review Letters, 98(15):156103.

Reis et al., 2010.Reis, P. M., Hure, J., Jung, S., Bush, J. W. M., and Clanet, C. (2010). Grabbing water. Soft Matter, 6(22):5705.

Rico-Guevara and Rubega, 2011.Rico-Guevara, A. and Rubega, M. A. (2011). The hummingbird tongue is a fluid trap, not a capillary tube. Proceedings of the National Academy of Sciences, 108(23):9356-9360.

Rivetti and Neukirch, 2012.Rivetti, M. and Neukirch, S. (2012). Instabilities in a drop-strip system: a simplified model. Proceedings of the Royal Society A: Mathematical, Physical and Engineering Sciences, 468(2141):1304-1324.

Roca-Cusachs et al., 2005.Roca-Cusachs, P., Rico, F., Martínez, E., Toset, J., Farré, R., and Navajas, D. (2005). Stability of Microfabricated High Aspect Ratio Structures in Poly(dimethylsiloxane). Langmuir, 21(12):5542-5548.

Roman and Bico, 2010.Roman, B. and Bico, J. (2010). Elasto-capillarity: deforming an elastic structure with a liquid droplet. Journal of Physics: Condensed Matter, 22(49):493101.

Rusanov, 1978.Rusanov, A. (1978). On the thermodynamics of deformable solid surfaces. Journal of Colloid and Interface Science, 63(2):330-345.

Salez et al., 2013.Salez, T., Benzaquen, M., and Raphaël, É. (2013). From adhesion to wetting of a soft particle. Soft Matter, 9(45):10699.

Schroll et al., 2013.Schroll, R. D., Adda-Bedia, M., Cerda, E., Huang, J., Menon, N., Russell, T. P., Toga, K. B., Vella, D., and Davidovitch, B. (2013). Capillary Deformations of Bendable Films. Physical Review Letters, 111(1):014301.

Schulman and Dalnoki-Veress, 2015.Schulman, R. D. and Dalnoki-Veress, K. (2015). Liquid Droplets on a Highly Deformable Membrane. Physical Review Letters, 115(20):206101.

Schulman et al., 2017.Schulman, R. D., Porat, A., Charlesworth, K., Fortais, A., Salez, T., Raphaël, E., and Dalnoki-Veress, K. (2017). Elastocapillary bending of microfibers around liquid droplets. Soft Matter, 13(4):720-724.

Shanahan, 1987.Shanahan, M. E. R. (1987). The influence of solid micro-deformation on contact angle equilibrium. Journal of Physics D: Applied Physics, 20(7):945-950.

Shanahan, 1988.Shanahan, M. E. R. (1988). The spreading dynamics of a liquid drop on a viscoelastic solid. Journal of Physics D: Applied Physics, 21(6):981-985.

Sharp et al., 2004.Sharp, K. G., Blackman, G. S., Glassmaker, N. J., Jagota, A., and Hui, C. Y. (2004). Effect of stamp deformation on the quality of microcontact printing: Theory and experiment. Langmuir, 20(15):6430-6438.

Singh et al., 2014.Singh, K., Lister, J. R., and Vella, D. (2014). A fluid-mechanical model of elastocapillary coalescence. Journal of Fluid Mechanics, 745:621-646.

Snoeijer, 2016.Snoeijer, J. H. (2016). Analogies between elastic and capillary interfaces. Physical Review Fluids, 1(6):060506.

Sokuler et al., 2010.Sokuler, M., Auernhammer, G. K., Roth, M., Liu, C., Bonacurrso, E., and Butt, H.-J. (2010). The Softer the Better: Fast Condensation on Soft Surfaces. Langmuir, 26(3):1544-1547.

Style et al., 2013a.Style, R. W., Boltyanskiy, R., Che, Y., Wettlaufer, J. S., Wilen, L. A., and Dufresne, E. R. (2013a). Universal Deformation of Soft Substrates Near a Contact Line and the Direct Measurement of Solid Surface Stresses. Physical Review Letters, 110(6):066103.

Style et al., 2013b.Style, R. W., Che, Y., Park, S. J., Weon, B. M., Je, J. H., Hyland, C., German, G. K., Power, M. P., Wilen, L. A., Wettlaufer, J. S., and Dufresne, E. R. (2013b). Patterning droplets with durotaxis. Proceedings of the National Academy of Sciences, 110(31):12541-12544.

Style and Dufresne, 2012.Style, R. W. and Dufresne, E. R. (2012). Static wetting on deformable substrates, from liquids to soft solids. Soft Matter, 8(27):7177.

Style et al., 2013c.Style, R. W., Hyland, C., Boltyanskiy, R., Wettlaufer, J. S., and Dufresne, E. R. (2013c). Surface tension and contact with soft elastic solids. Nature Communications, 4:2728. 
Style et al., 2017.Style, R. W., Jagota, A., Hui, C.-Y., and Dufresne, E. R. (2017). Elastocapillarity: Surface Tension and the Mechanics of Soft Solids. Annual Review of Condensed Matter Physics, 8(1):annurev-conmatphys-031016-025326.

Style et al., 2015.Style, R. W., Wettlaufer, J. S., and Dufresne, E. R. (2015). Surface tension and the mechanics of liquid inclusions in compliant solids. Soft Matter, 11(4):672-679.

Syms et al., 2003.Syms, R., Yeatman, E., Bright, V., and Whitesides, G. (2003). Surface tensionpowered self-assembly of microstructures - The state-of-the-art. Journal of Microelectromechanical Systems, 12(4):387-417.

Taffetani and Ciarletta, 2015.Taffetani, M. and Ciarletta, P. (2015). Elastocapillarity can control the formation and the morphology of beads-on-string structures in solid fibers. Physical Review E, 91(3):032413.

Taroni and Vella, 2012.Taroni, M. and Vella, D. (2012). Multiple equilibria in a simple elastocapillary system. Journal of Fluid Mechanics, 712:273-294.

Tas et al., 1996.Tas, N., Sonnenberg, T., Jansen, H., Legtenberg, R., and Elwenspoek, M. (1996). Stiction in surface micromachining. Journal of Micromechanics and Microengineering, 6(4):385397.

Tawfick et al., 2016.Tawfick, S. H., Bico, J., and Barcelo, S. (2016). Three-dimensional lithography by elasto-capillary engineering of filamentary materials. MRS Bulletin, 41(02):108-114.

Taylor, 1963.Taylor, G. (1963). On the shapes of parachutes. In Batchelor, G. K., editor, The Scientific Papers of Sir Geoffrey Ingram Taylor Vol. 3, pages 26-37. Cambridge Univ. Press.

Toga et al., 2013.Toga, K. B., Huang, J., Cunningham, K., Russell, T. P., and Menon, N. (2013). A drop on a floating sheet: boundary conditions, topography and formation of wrinkles. Soft Matter, 9(34):8289.

Vollrath and Edmonds, 1989.Vollrath, F. and Edmonds, D. T. (1989). Modulation of the mechanical properties of spider silk by coating with water. Nature, 340(6231):305-307.

Wang et al., 2016.Wang, K.-y., Chou, C.-h., Liao, C.-y., Li, Y.-r., and Cheng, H.-c. (2016). Densification effects of the carbon nanotube pillar array on field-emission properties. Japanese Journal of Applied Physics, 55(6S1):06GF12.

Wei and Mahadevan, 2014.Wei, Z. and Mahadevan, L. (2014). Continuum dynamics of elastocapillary coalescence and arrest. EPL (Europhysics Letters), 106(1):14002.

Weijs et al., 2013.Weijs, J. H., Andreotti, B., and Snoeijer, J. H. (2013). Elasto-capillarity at the nanoscale: on the coupling between elasticity and surface energy in soft solids. Soft Matter, $9(35): 8494$.

Weijs et al., 2014.Weijs, J. H., Snoeijer, J. H., and Andreotti, B. (2014). Capillarity of soft amorphous solids: A microscopic model for surface stress. Physical Review E, 89(4):042408.

Wexler et al., 2014.Wexler, J. S., Heard, T. M., and Stone, H. A. (2014). Capillary Bridges between Soft Substrates. Physical Review Letters, 112(6):066102.

Xuan and Biggins, 2016.Xuan, C. and Biggins, J. (2016). Finite-wavelength surface-tension-driven instabilities in soft solids, including instability in a cylindrical channel through an elastic solid. Physical Review E, 94(2):023107.

Zell et al., 2010.Zell, Z. A., Choi, S. Q., Leal, L. G., and Squires, T. M. (2010). Microfabricated deflection tensiometers for insoluble surfactants. Applied Physics Letters, 97(13):1-3.

Zhao and Fan, 2006.Zhao, Y.-P. and Fan, J.-G. (2006). Clusters of bundled nanorods in nanocarpet effect. Applied Physics Letters, 88(10):103123. 